\title{
repisälud
}

This is the peer reviewed version of the following article:

Lim, R., Sugino, T., Nolte, H., Andrade, J., Zimmermann, B., Shi, C., . . Potente, M. (2019). Deubiquitinase USP10 regulates Notch signaling in the endothelium. Science, 364(6436), 188-193. doi:10.1126/science.aat0778

which has been published in final form at: https://doi.org/10.1126/science.aat0778 


\section{Title: Deubiquitinase USP10 regulates Notch signaling in the endothelium}

Authors: R. Lim ${ }^{1 \S}$, T. Sugino ${ }^{1 \S}$, H. Nolte ${ }^{2}$, J. Andrade ${ }^{1}$, B. Zimmermann ${ }^{1}$, C. Shi ${ }^{1}$, A.

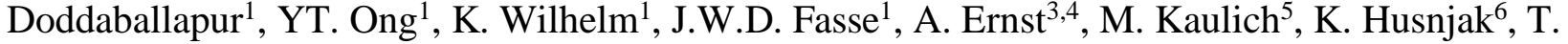
Boettger $^{7}$, S. Guenther ${ }^{7}$, T. Braun ${ }^{7}$, M. Krüger ${ }^{2}$, R. Benedito ${ }^{8}$, I. Dikic ${ }^{9}$, M. Potente ${ }^{1,10^{*}}$

\section{Affiliations:}

${ }^{1}$ Angiogenesis \& Metabolism Laboratory, Max Planck Institute for Heart and Lung Research, D61231 Bad Nauheim, Germany

${ }^{2}$ Institute for Genetics and Excellence Cluster on Cellular Stress Responses in Aging-Associated Diseases (CECAD), University of Cologne, D-50931 Cologne, Germany

${ }^{3}$ Protein Engineering Group, Institute of Biochemistry II, Goethe University, D-60590 Frankfurt (Main), Germany

${ }^{4}$ Fraunhofer Institute for Molecular Biology and Applied Ecology IME, Project Group Translational Medicine and Pharmacology TMP, D-60590 Frankfurt (Main), Germany.

${ }^{5}$ Gene Editing Group, Institute of Biochemistry II, Goethe University, D-60590 Frankfurt (Main), Germany

${ }^{6}$ Ubiquitin Signaling Group, Institute of Biochemistry II, Goethe University, D-60590 Frankfurt (Main), Germany

${ }^{7}$ Department of Cardiac Development and Remodeling, Max Planck Institute for Heart and Lung Research, D-61231 Bad Nauheim, Germany

${ }^{8}$ Molecular Genetics of Angiogenesis Laboratory, Centro Nacional de Investigaciones Cardiovasculares (CNIC), Madrid 28029, Spain

${ }^{9}$ Molecular Signaling Group, Institute of Biochemistry II, Goethe University, D-60590 Frankfurt (Main), Germany

${ }^{10}$ DZHK (German Center for Cardiovascular Research), partner site Frankfurt Rhine-Main, D13347 Berlin, Germany

$\S_{\text {Contributed equally }}$

*Correspondence to: Michael Potente (michael.potente@ mpi-bn.mpg.de) 


\begin{abstract}
Notch signaling is a core patterning module for vascular morphogenesis, which codetermines the sprouting behaviour of endothelial cells (ECs). Tight quantitative and temporal control of Notch activity is essential for vascular development, yet the details of Notch regulation in ECs are incompletely understood. We found that ubiquitin-specific peptidase 10 (USP10) interacted with the NOTCH1 intracellular domain (NICD1) to slow the ubiquitin-dependent turnover of this short-lived form of the activated NOTCH1 receptor. Accordingly, inactivation of USP10 reduced NICD1 abundance and stability, and diminished Notch-induced target gene expression in ECs. In mice, loss of endothelial Usp10 increased vessel sprouting and partially restored the patterning defects caused by ectopic expression of NICD1. Thus, USP10 functions as an NICD1 deubiquitinase, which fine-tunes endothelial Notch responses during angiogenic sprouting.
\end{abstract}

One Sentence Summary: USP10 regulates endothelial Notch signaling. 
Main Text: To add new vessel sprouts to an existing vascular network, ECs specialize into leading tip cells and following stalk cells (1), which dynamically interchange positions (2). Signaling by the delta-like 4 (DLL4) ligand and NOTCH1 receptor helps to coordinate these processes. Its inhibition causes excessive sprouting and vessel overgrowth, whereas forced activation leads to vascular rarefaction (3-10). DLL4 induces the cleavage of NOTCH1 by the $\gamma$-secretase protease, which releases the NICD1. This fragment forms a ternary complex with the DNA binding protein recombining binding protein suppressor of hairless (RBPJ) and the co-activators mastermind-like protein 1-3 (MAML1-3) to drive transcription of Notch target genes (11-13). In many cellular contexts, the half-life of NICD1 is short because it undergoes rapid proteasomal degradation mediated by ubiquitin ligases such as SKP1-CUL1-F-box protein complex (SCF) containing the substrate recognition component F-box and WD repeat domain containing 7 (FBXW7) (14-16). Inactivation of FBXW7 increases the abundance of NICD1 levels and causes severe defects in vascular sprouting (17-19). Having the right amount of NICD1 at the right time, is thus critical for the cooperative behavior of ECs.

To identify regulators of NICD1, we examined the NICD1 interactome in human embryonic kidney (HEK293T) cells using stable isotope labeling with amino acids in cell culture (SILAC)-based quantitative proteomics (Fig. S1A). LC-MS/MS analysis revealed that NICD1 interacted with core components of the Notch transcription complex including RBPJ and MAML1 (Fig. 1A). USP10, a deubiquitinase that removes ubiquitin modifications from its protein targets, was also among the NICD1-immunoprecipitated proteins (Fig. 1, A and B). To confirm this, we used primary ECs, in which USP10 is an abundant nucleo-cytoplasmic protein (Fig. 1, C and D; Fig. S1, B-D). We immunoprecipitated NICD1 from cultured human umbilical vein endothelial cells (HUVECs) that expressed a V5 epitope-tagged NICD1 (NICD1 ${ }^{\mathrm{V} 5}$ ). Immunoblotting for 
USP10 demonstrated that endogenous USP10 associated with $\mathrm{NICD} 1^{\mathrm{V} 5}$, as did the ternary complex factors RBPJ and MAML1 (Fig. 1E). This interaction also occurred when endogenous NICD1 was immunoprecipitated from HUVECs demonstrated by a DLL4-inducible NICD1USP10 protein complex that is enriched in the nuclear fraction (Fig. 1F; Fig. S1E). Together, these findings suggest that USP10 interacts with NICD1 in ECs.

To investigate the role of USP10 in endothelial Notch signaling, we performed knockdown experiments in HUVECs using small interfering RNAs (siRNAs) and assayed Notch signaling with various Notch-responsive luciferase reporters. Treatment of scrambled siRNA (siCtrl)transfected HUVECs with DLL4 induced a $\gamma$-secretase inhibitor (dibenzazepine, DBZ)-sensitive and RBPJ-dependent increase in Notch reporter gene activity (Fig. 2, A and B; Fig. S2B). Depletion of USP10 (siUSP10) did not affect the baseline activity of the reporters but reduced the DLL4-induced increase of Notch activity (Fig. 2, A and B; Fig. S2, A and B), suggesting that USP10 regulates canonical Notch responses in ECs. These findings were corroborated by a genome-wide transcriptomic analysis of siCtrl- and siUSP10-transfected HUVECs stimulated with DLL4 or unstimulated for 24 hours, revealing that depletion of USP10 reduced the expression of canonical Notch target genes (e.g. Hes related family BHLH transcription factor with YRPW motif 1 (HEY1), HEY2, Hes family BHLH transcription factor 1 (HES1), Notch regulated Ankyrin repeat protein $(N R A R P))$ as well as other genes that are regulated by DLL4-Notch (20) (Fig. 2C; Fig. S2C). Quantitative real-time PCR (qRT-PCR) analysis confirmed this finding (Fig. S2, D and E).

Loss of USP10 did not lead to compensatory expression changes of other deubiquitinases (Fig. S2A), nor did we detect expression changes of core Notch signaling components in unstimulated HUVECs that might explain reduction in pathway activity (Fig. S2F). We surmised that USP10 regulates Notch signaling post-transcriptionally and focused on the short-lived NICD1 
protein. Immunoblot analysis showed that NICD1 protein was low under basal conditions but became abundant in response to short (6 hours) or prolonged (24 hours) stimulation with DLL4 (Fig. 2D; Fig. S3A). Inactivation of USP10 by siRNA or CRISPR/Cas9 gene editing reduced the abundance of NICD1 in response to DLL4 stimulation without affecting basal NOTCH1 mRNA expression (Fig. 2, D-F; Fig. S3, A-D). Similar results were obtained with the Notch ligands Deltalike 1 (DLL1) or Jagged 1 (JAG1), which trigger distinct Notch responses and signaling dynamics $(8,21)$. However, DLL1 and JAG1, in comparison to DLL4, were weaker NOTCH1 agonists in HUVECs and higher concentrations were needed for receptor activation (Fig. S3, E-G). Depletion of USP7, USP11, or USP15, three USP family members that associate with NICD1 in cancer cells (22), did not change NICD1 protein expression (Fig. S4A). Thus, USP10 regulates endothelial Notch signaling by controlling NICD1 abundance, though we cannot exclude that alternative mechanisms may contribute to the observed effects on the Notch pathway.

This regulation appears to occur at the level of the NICD1 (downstream of $\gamma$-secretase cleavage) because the amounts of ectopically expressed NICD1 ${ }^{\mathrm{V} 5}$ were also reduced in USP10deficient ECs (Fig. S4B). Overexpression of USP10, on the other hand, enhanced abundance of NICD1 in HUVECs (Fig. 2G; Fig. S4C). Because USP10 is a deubiquitinase, we tested whether USP10 interferes with NICD1 protein degradation. Indeed, treatment of ECs with the proteasome inhibitor MG132 or depletion of FBXW7, both increased abundance of NICD1 protein in USP10deficient HUVECs (Fig. S5, A and B). Moreover, measurement of the rate of NICD1 decay in cycloheximide-treated cells showed that the half-life of endogenous NICD1 protein was decreased upon depletion of USP10 (Fig. 3A). Depletion of endothelial USP10 did not alter the abundance of cellular tumor antigen p53 (p53) or sirtuin 6 (SIRT6) (Fig. S5C), two substrates of USP10 in 
tumor cells $(23,24)$, suggesting that the signaling context in primary ECs differs from that in cancer cell lines.

To test whether USP10 modulates NICD1 ubiquitination in ECs, we captured ubiquitinated proteins from HUVECs using Halo-tagged tandem ubiquitin binding entities (TUBE ${ }^{\text {Halo }}$ ) (25) followed by immunoblotting with an NICD1 antibody. This analysis revealed a high molecular size ubiquitin smear in DLL4-stimulated ECs that was enhanced by MG132 treatment (Fig. S5D). Co-expression of USP10 decreased this ubiquitin signal (Fig. S5E), suggesting that USP10 can deubiquitinate NICD1. Consistent with these data, depletion of USP10 in DLL4-stimulated HUVECs enhanced endogenous ubiquitination of NICD1 (Fig. 3B). This increase in NICD1 ubiquitination was observed in the nuclear fraction of HUVECs (Fig. 3C), where NICD1 is targeted by ubiquitin ligases such as $\operatorname{SCF}^{\mathrm{FBXW7}}$ (14-16), and where NICD1 and USP10 interact. To finally assess whether USP10 deubiquitinates NICD1, we purified ubiquitinated NICD1 from HEK293T cells that were co-transfected with NICD1 ${ }^{\mathrm{V} 5}$, HA-tagged ubiquitin $\left(\mathrm{Ub}^{\mathrm{HA}}\right)$ and FLAGtagged FBXW7 (FBXW7 ${ }^{\mathrm{FLAG}}$ ) (Fig. S5F). Incubation of ubiquitinated NICD1 with recombinant USP10 under conditions allowing NICD1 deubiquitination by the promiscuous catalytic core of USP2 led to decreased poly-ubiquitination of NICD1 (Fig. 3D), suggesting that USP10 functions as a NICD1 deubiquitinase.

To understand the physiological role of USP10 in the regulation of Notch signaling, we generated mice, in which exons 7 and 8 of $U s p 10$ were flanked by loxP sites (Usp10 flffl) (Fig. S6A). Germline deletion of Usp10 (Usp10-/) caused perinatal lethality (Fig. S6B) (26). To bypass lethality, we crossed $U s p 10^{f l f l}$ mice with a tamoxifen-inducible cre deleter (creERT2) that is driven by the endothelial-selective platelet-derived growth factor subunit B (Pdgfb) promoter (PdgfbcreERT2 deleter) and assessed vascular phenotypes of the resulting mutants $\left(U s p 10^{i E C-K O}\right)$ in the 
mouse retina - a well-established model of Notch-dependent angiogenesis. We injected 4hydroxytamoxifen (4-OHT) from postnatal day (P) 1 to day 4 and analyzed retinal angiogenesis at P7 (Fig. S6C). Endothelial-restricted deletion of Usp10 increased EC density and sprouting at the angiogenic front, but had little or even opposite effects in the central parts of the retinal vasculature (Fig. 4, A and B). At the front, Usp10 $0^{i E C-K O}$ retinas displayed increased numbers of endothelial sprouts and filopodia (Fig. 4, C, D and F), and showed enhanced immunostaining for endothelial cell-specific molecule 1 (ESM1) (Fig. 4E; Fig. S6D), a tip cell marker that is suppressed by DLL4Notch signaling (Fig. S2C) (9, 27). In Usp10 mutant mice, ECs accumulated in veins and perivenous capillaries, and veins were enlarged (Fig. S6, D and E). Together, these phenotypes resemble aspects of reduced DLL4-NOTCH1 signaling in the retinal endothelium $(3,5,6,9)$ and indicate that USP10 deficiency reduces Notch responses in vivo.

We tested whether USP10 inactivation would restore endothelial sprouting behavior when Notch signaling was aberrantly activated. We used a cre-inducible Notch gain-of-function mouse model, in which full-length NICD1 is expressed from the ubiquitously active CMV early enhancer/chicken $\beta$ actin $(C A G)$ promoter (Fig. S7A). Expression of NICD1 (as well as membrane-targeted Tomato and nuclear-targeted green fluorescent protein $(G F P)$ is controlled by a floxed STOP cassette, which can be removed by cre-mediated excision (Rosa26 $\left.6^{N I C D I}\right)(\mathrm{S} 7, \mathrm{~A}$ and B). Pdgfb-creERT2-mediated recombination in $R o s a 26^{N I C D 1}\left(N I C D 1^{i E C-O E}\right)$ mice led to severe retinal vascular defects, which correlated with the expression of the fluorescent reporters (Fig. 4, $\mathrm{G}$ and $\mathrm{H}$; Fig. S7B). The vascular network in $N I C D 1^{i E C-O E}$ mutant mice was sparse and had fewer vessel sprouts (Fig. 4, G and H; Fig. 7, C and D), a phenotype that is consistent with increased endothelial Notch activity $(9,19)$. We also combined Rosa26 $6^{N I C D 1}, U s p 10^{f l o x}$ and Pdgfb-creERT2 alleles $\left(N I C D 1^{i E C-O E} ; U s p 10^{i E C-K O}\right)$ to generate compound mutants. Deletion of Usp10 reduced the 
sprouting defects caused by forced endothelial expression of NICD1 (Fig. 4, I and J). Compared to the vasculature in the $N I C D 1^{i E C-O E}$ mice, $N I C D 1^{i E C-O E} ;$ Usp $10^{i E C-K O}$ double-mutants had an increased number of vessel sprouts and branches giving rise to a more dense vascular network at the angiogenic front (Fig. 4, I and J). Thus, USP10 deficiency can, at least in part, restore vessel density in conditions of enhanced NICD1 signaling, suggesting that USP10 is a regulator of Notchdependent vascular morphogenesis in vivo.

In summary, we identify USP10-mediated deubiquitination of NICD1 as a mechanism to delay the rate of its degradation, which prolongs cellular Notch responses. Such regulation appears to be particularly relevant for angiogenic sprouting during which dynamic changes in Notch activity determine specification and position of tip and stalk cells (28). Importantly, USP10 is itself a regulated protein and recent studies reported that AMP-activated kinase (AMPK)-mediated phosphorylation activates the enzyme (29). Because AMPK senses cellular energy levels and is activated by hypoxia and nutrient limitation, USP10 may be particularly active in ECs that face oxygen- and nutrient-deprivation, as they do when invading avascular tissues. Indeed, we observed increased USP10 activity in ECs that were cultured in hypoxic or nutrient-starved conditions (Fig. S8, A and B). These considerations could explain why the loss of USP10 in mice causes Notch loss-of-function phenotypes only at the sprouting front and not in the central plexus, where ECs are exposed to physiological oxygen and nutrient levels. Further studies on the regulation of USP10 may reveal in which other cell types and (patho-)physiological contexts the USP10dependent modulation of Notch signaling is at play (30). 


\section{References:}

1. M. Potente, H. Gerhardt, P. Carmeliet, Basic and therapeutic aspects of angiogenesis. Cell 146, 873-887 (2011).

2. L. Jakobsson et al., Endothelial cells dynamically compete for the tip cell position during angiogenic sprouting. Nat Cell Biol 12, 943-953 (2010).

3. M. Hellstrom et al., Dll4 signalling through Notch1 regulates formation of tip cells during angiogenesis. Nature 445, 776-780 (2007).

4. A. F. Siekmann, N. D. Lawson, Notch signalling limits angiogenic cell behaviour in developing zebrafish arteries. Nature 445, 781-784 (2007).

5. S. Suchting et al., The Notch ligand Delta-like 4 negatively regulates endothelial tip cell formation and vessel branching. Proc Natl Acad Sci U S A 104, 3225-3230 (2007).

6. I. B. Lobov et al., Delta-like ligand 4 (Dl14) is induced by VEGF as a negative regulator of angiogenic sprouting. Proc Natl Acad Sci U S A 104, 3219-3224 (2007).

7. J. D. Leslie et al., Endothelial signalling by the Notch ligand Delta-like 4 restricts angiogenesis. Development 134, 839-844 (2007).

8. R. Benedito et al., The notch ligands Dll4 and Jagged1 have opposing effects on angiogenesis. Cell 137, 1124-1135 (2009).

9. M. E. Pitulescu et al., Dl14 and Notch signalling couples sprouting angiogenesis and artery formation. Nat Cell Biol 19, 915-927 (2017).

10. S. S. Hasan et al., Endothelial Notch signalling limits angiogenesis via control of artery formation. Nat Cell Biol 19, 928-940 (2017).

11. C. Siebel, U. Lendahl, Notch Signaling in Development, Tissue Homeostasis, and Disease. Physiological Reviews 97, 1235-1294 (2017).

12. S. J. Bray, Notch signalling in context. Nature Reviews Molecular Cell Biology 17, 722735 (2016).

13. R. Kopan, M. X. Ilagan, The canonical Notch signaling pathway: unfolding the activation mechanism. Cell 137, 216-233 (2009).

14. C. Oberg et al., The Notch intracellular domain is ubiquitinated and negatively regulated by the mammalian Sel-10 homolog. The Journal of Biological Chemistry 276, 3584735853 (2001).

15. C. J. Fryer, J. B. White, K. A. Jones, Mastermind recruits CycC:CDK8 to phosphorylate the Notch ICD and coordinate activation with turnover. Molecular Cell 16, 509-520 (2004).

16. J. O'Neil et al., FBW7 mutations in leukemic cells mediate NOTCH pathway activation and resistance to gamma-secretase inhibitors. J Exp Med 204, 1813-1824 (2007).

17. M. T. Tetzlaff et al., Defective cardiovascular development and elevated cyclin E and Notch proteins in mice lacking the Fbw7 F-box protein. Proc Natl Acad Sci U S A 101, 3338-3345 (2004). 
18. R. Tsunematsu et al., Mouse Fbw7/Sel-10/Cdc4 is required for notch degradation during vascular development. The Journal of Biological Chemistry 279, 9417-9423 (2004).

19. N. Izumi et al., Fbxw7 controls angiogenesis by regulating endothelial Notch activity. PLoS One 7, e41116 (2012).

20. L. S. Harrington et al., Regulation of multiple angiogenic pathways by D114 and Notch in human umbilical vein endothelial cells. Microvasc Res 75, 144-154 (2008).

21. N. Nandagopal et al., Dynamic Ligand Discrimination in the Notch Signaling Pathway. Cell 172, 869-880.e819 (2018).

22. A. Yatim et al., NOTCH1 nuclear interactome reveals key regulators of its transcriptional activity and oncogenic function. Molecular Cell 48, 445-458 (2012).

23. J. Yuan, K. Luo, L. Zhang, J. C. Cheville, Z. Lou, USP10 regulates p53 localization and stability by deubiquitinating p53. Cell 140, 384-396 (2010).

24. Z. Lin et al., USP10 antagonizes c-Myc transcriptional activation through SIRT6 stabilization to suppress tumor formation. Cell Rep 5, 1639-1649 (2013).

25. R. Hjerpe et al., Efficient protection and isolation of ubiquitylated proteins using tandem ubiquitin-binding entities. EMBO Reports 10, 1250-1258 (2009).

26. M. Higuchi et al., USP10 Is an Essential Deubiquitinase for Hematopoiesis and Inhibits Apoptosis of Long-Term Hematopoietic Stem Cells. Stem Cell Reports 7, 1116-1129 (2016).

27. S. F. Rocha et al., Esm1 modulates endothelial tip cell behavior and vascular permeability by enhancing VEGF bioavailability. Circ Res 115, 581-590 (2014).

28. B. Ubezio et al., Synchronization of endothelial Dll4-Notch dynamics switch blood vessels from branching to expansion. Elife 5, (2016).

29. M. Deng et al., Deubiquitination and Activation of AMPK by USP10. Molecular Cell 61, 614-624 (2016).

30. J. Zhang, M. Liu, Y. Su, J. Du, A. J. Zhu, A targeted in vivo RNAi screen reveals deubiquitinases as new regulators of Notch signaling. G3 (Bethesda, Md.) 2, 1563-1575 (2012). 
Acknowledgments: We thank Jian Yuan, Zhenkun Lou and Nikita Popov for expression plasmids, and Mads Gyrd-Hansen for advice on the TUBE assays. This work is supported by the Max Planck Society, the European Research Council (ERC) Starting Grant ANGIOMET (311546), the ERC Consolidator EMERGE (773047), the Deutsche Forschungsgemeinschaft (SFB 834), the Excellence Cluster Cardiopulmonary System (EXC 147/1), the LOEWE grant Ub-Net, the DZHK (German Center for Cardiovascular Research), the Stiftung Charité, and the European Molecular Biology Organization (EMBO) Young Investigator Programme.

Author contributions: R.L., T.S., H.N., J.A., B.Z., C.S., A.D., Y.T.O., K.W., J.W.D.F., A.E., M.Ka., K.H., T.Bo., and S.G. performed experiments. R.L., T.S., H.N., J.A., B.Z., C.S., A.D., Y.T.O., K.W., J.W.D.F., A.E., M.Ka., K.H., T.Bo., S.G and M.P. analysed data. A.E., M.Ka., K.H., T.Br., M.Kr., R.B., and I.D. provided essential reagents and protocols. M.P. supervised the study. R.L., T.S., I.D. and M.P. wrote the paper. All authors discussed the results and commented on the manuscript.

Competing financial interests: The authors declare no competing financial interests.

Author information: Correspondence and requests for materials should be addressed to M.P. (michael.potente@mpi-bn.mpg.de). 


\section{Figures:}

\section{Fig. 1. USP10 interacts with NICD1 in ECs.}

A, 2D plot with $\log _{2} \mathrm{H} / \mathrm{L}$ SILAC ratios of the quantified proteins, correlating to an enrichment in NICD1-IP versus Control-IP on the x axis, and signal intensity (log scale) in arbitrary units on the y axis. B, MS/MS spectra of light-labeled (grey) and heavy-labeled (red) peptides separated by 8 Da, showing doubly charged EPLGEDSVGLKPLK and EGLVPVSEDPVAIK peptides from NICD1 and USP10, respectively. C, Immunoblot analysis of USP10 protein expression in several EC types. HAEC, human aortic endothelial cells. HMVEC, human micro-vascular endothelial cells. HLEC, human lymphatic endothelial cells. VEGFR2, vascular endothelial growth factor

receptor 2. D, USP10 protein expression in cytoplasmic and nuclear fractions isolated from control or DLL4-stimulated HUVECs. Histone H3 and Tubulin were used as nuclear and cytoplasmic markers, respectively. E, Co-immunoprecipitation of adenovirus-delivered NICD1-V5 and endogenous USP10, MAML1 and RBPJ from HUVECs. F, Co-immunoprecipitation of NICD1 and USP10 from HUVECs treated with or without DLL4 for $24 \mathrm{~h}$. Endogenous NICD1 was immunoprecipitated by anti-NOTCH1 antibody.

\section{Fig. 2. USP10 deficiency reduces endothelial Notch signaling.}

A, Activation of TP1-driven luciferase (luc) reporter in scrambled-control (siCtrl)- or USP10 siRNA (siUSP10)-transfected HUVECs after treatment with vehicle or delta-like 4 (DLL4), in the presence or absence of a $\gamma$-secretase inhibitor (DBZ) for $24 \mathrm{~h}$. B, Activation of 4xRBPJ- or 4xRBPJ mutant-( $\left.{ }^{\text {mut }}\right)$ luc reporter in siCtrl- or siUSP10-transfected HUVECs after treatment with vehicle or DLL4 for 24 h. C, Heat map of differentially expressed genes in siCtrl- or siUSP10-transfected 
HUVECs treated with vehicle or DLL4 for $24 \mathrm{~h}$. Data were obtained by microarray analysis performed in triplicate for each condition. Differentially regulated genes with a cut-off P value of $<0.05$ and a fold-change of $>2.0$ (siCtrl vs. siCtrl + DLL4) are shown. D, Immunoblot analysis of NICD1 expression in siCtrl- or siUSP10-transfected HUVECs stimulated with DLL4 or vehicle for 6 and 24 h. E, qRT-PCR analysis of NOTCH1 mRNA expression in control and USP10 knockdown HUVECs. Values are represented relative to expression of $\beta$-ACTIN. F, NICD1 levels in control and USP10 knockout ECs generated by lentiviral transduction of HUVECs with control (gCtrl) or USP10 (gUSP10)-targeting gRNAs as well as a FLAG-tagged CAS9 nuclease. HUVECs stimulated with vehicle or DLL4 for $24 \mathrm{~h}$. G, NICD1 immunoblot analysis from HUVECs transduced with a control (LentiCtrl) or a GFP-tagged USP10 encoding lentivirus (LentiUSP10 ${ }^{\mathrm{GFP}}$ ). NICD1 abundance was determined after $24 \mathrm{~h}$ of DLL4 stimulation. Error bars: mean $\pm \mathrm{SEM}$ of $\mathrm{n}>3$. $* * * P<0.001, * * * * P<0.0001, \mathrm{NS}$, not significant.

\section{Fig. 3. USP10 regulates NICD1 stability by altering its ubiquitin-dependent turnover.}

A, NICD1 abundance in HUVECs transfected with siCtrl or siUSP10. After $24 \mathrm{~h}$, cells were stimulated with DLL4 after pre-treatment with cycloheximide (CHX). ECs were collected at 1hour intervals and NICD1 protein levels analyzed by immunoblotting. Fold-change in NICD1 levels is shown on the right. B, NICD1 ubiquitination pattern in control (gCtrl) or USP10 knockout (gUSP10) HUVECs stimulated with vehicle or DLL4 for $24 \mathrm{~h}$. Cell lysates were incubated with Halo-tagged tandem ubiquitin binding entities (HaloTUBE) to enrich ubiquitinated proteins. Ubiquitinated NICD1 was detected by immunoblotting using an NICD1-specific antibody. C, Endogenous NICD1 ubiquitination in cytoplasmic (Cyto) and nuclear (Nuc) fractions derived from control (gCtrl) or USP10 knockout (gUSP10) HUVECs stimulated with vehicle or DLL4 for $24 \mathrm{~h}$. 
D, Ubiquitinated $\mathrm{NICD}^{\mathrm{V} 5}$ was purified by anti-V5 immunoaffinity chromatography from HEK293T cells transfected with NICD1 $1^{\mathrm{V} 5}, \mathrm{FBXW} 7^{\mathrm{FLAG}}$ and HA-ubiquitin $\left(\mathrm{Ub}^{\mathrm{HA}}\right)$ and then incubated in vitro with vehicle, recombinant USP10 or the recombinant catalytic core of USP2. Levels of NICD1 ubiquitination were determined by immunoblotting for HA. Error bars: mean \pm SEM of $\mathrm{n}>3 . * * * P<0.001, * * * * P<0.0001$.

Fig. 4. Deletion of Usp10 in ECs increases angiogenic sprouting and partially restores vascular defects caused by forced NICD1 expression.

A, Immunofluorescence staining for PECAM in P7 mouse retinas of 4-OHT-injected control $\left(U s p 10^{f l f t}\right)$ and $U s p 10^{i E C K O}\left(P d g f b\right.$-creERT2;Usp10 $\left.0^{f l f f}\right)$ mice. B, Images of PECAM- (red) and ERG- (cyan) stained P7 retinas of control and Usp10 ${ }^{i E C-K O}$ mutants showing clustering of ECs at the angiogenic front. $\mathbf{C}$, Higher magnification confocal images of PECAM-labeled P7 retinas isolated from control and Usp10 ${ }^{i E C-K O}$ mice. Endothelial filopodia are denoted by the small red dots. D, PECAM- (blue), ICAM2- (ICAM; green) and collagen IV (COL; red) labeling of control and $U s p 10^{i E C-K O}$ retinas at $\mathrm{P} 7$. The vasculature at the angiogenic front is shown. E, Confocal images showing PECAM (grey), ERG (red) and ESM1 (yellow) immunostaining of the retinal vasculature in control and Usp10 $0^{i E C-K O}$ mutants at P7. ESM1 immunostaining behind the angiogenic front was enhanced in $U s p 10^{i E C-K O}$ mice. F, Quantifications of vascular parameters at the angiogenic front in P7 control and mutant retinas as indicated. G, Confocal images of PECAMlabeled (grey) P6 retinas in control and NICDI ${ }^{i E C-O E}$ mice. $\mathbf{H}$, Immunofluorescence staining for PECAM- (blue), ICAM2- (ICAM; green) and collagen IV (COL; red) in a P6 mouse retina of 4OHT-injected control and NICDI $I^{i E C-O E}$ mice. I,J, Confocal images (I) of PECAM- (red) and ERG(cyan) labeled retinas in control, $N I C D I^{i E C-O E}$ and $N I C D I^{i E C-O E} ; U s p 10^{i E C-K O}$ double mutants. The 
quantification $(\mathrm{J})$ of the endothelial coverage at the angiogenic front in the respective genotypes is shown. Error bars: mean \pm SEM of $\mathrm{n}>3$. ${ }^{*} * \mathrm{P}<0.01, * * * * \mathrm{P}<0.0001$, NS, not significant. 


\section{Figure 1}

A

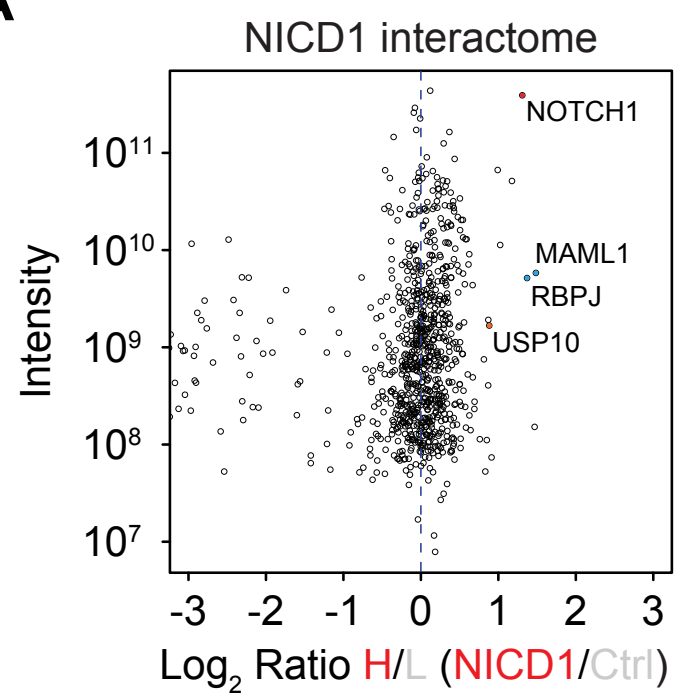

C

D
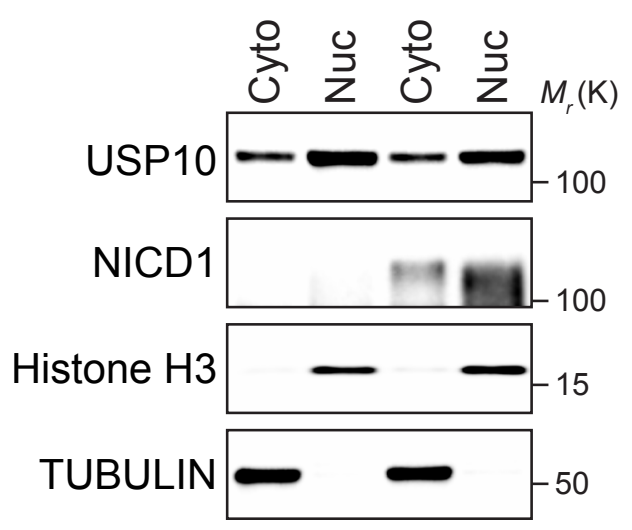

DLL4 - - + +
B
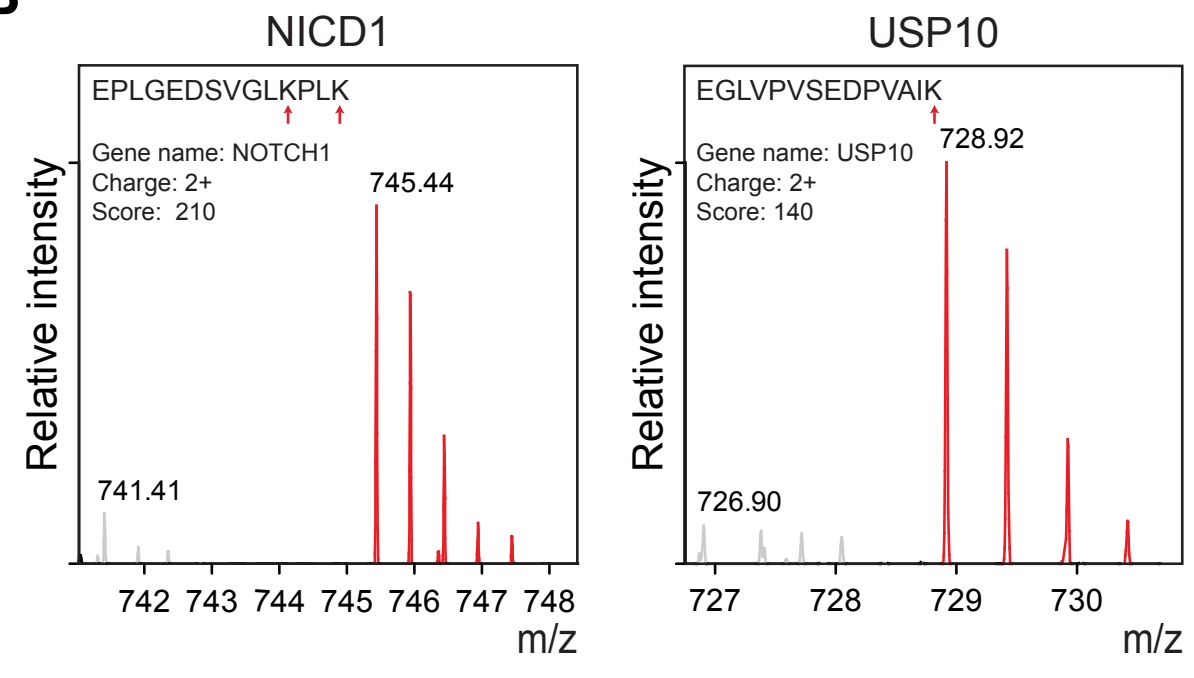

E

$\mathbf{F}$

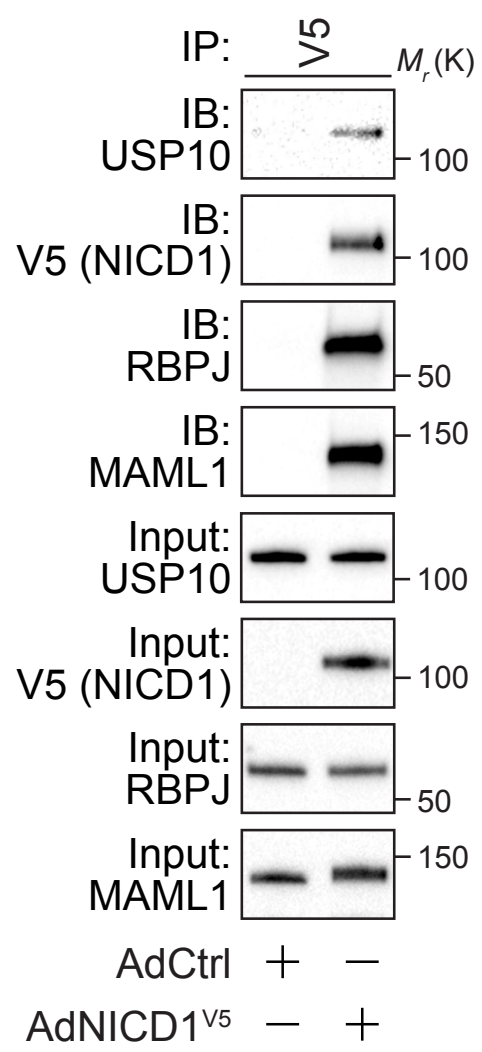

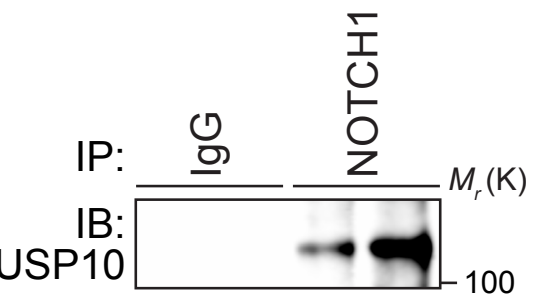
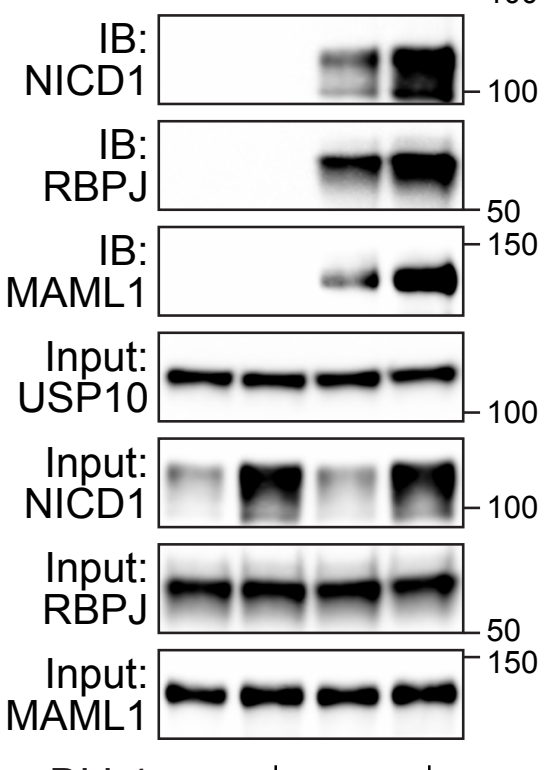
A

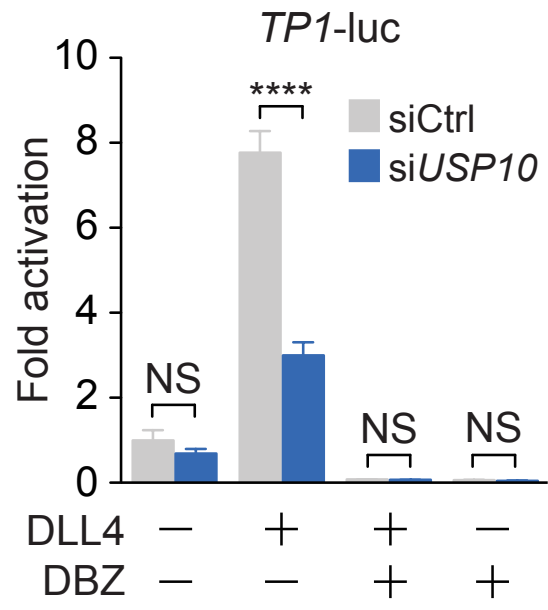

B

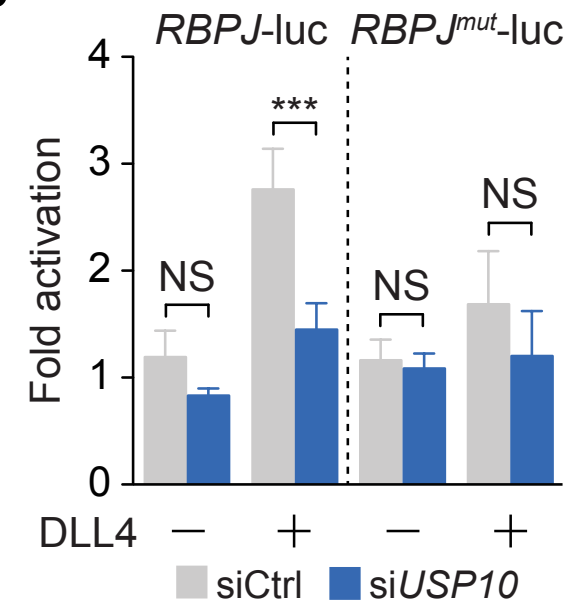

E

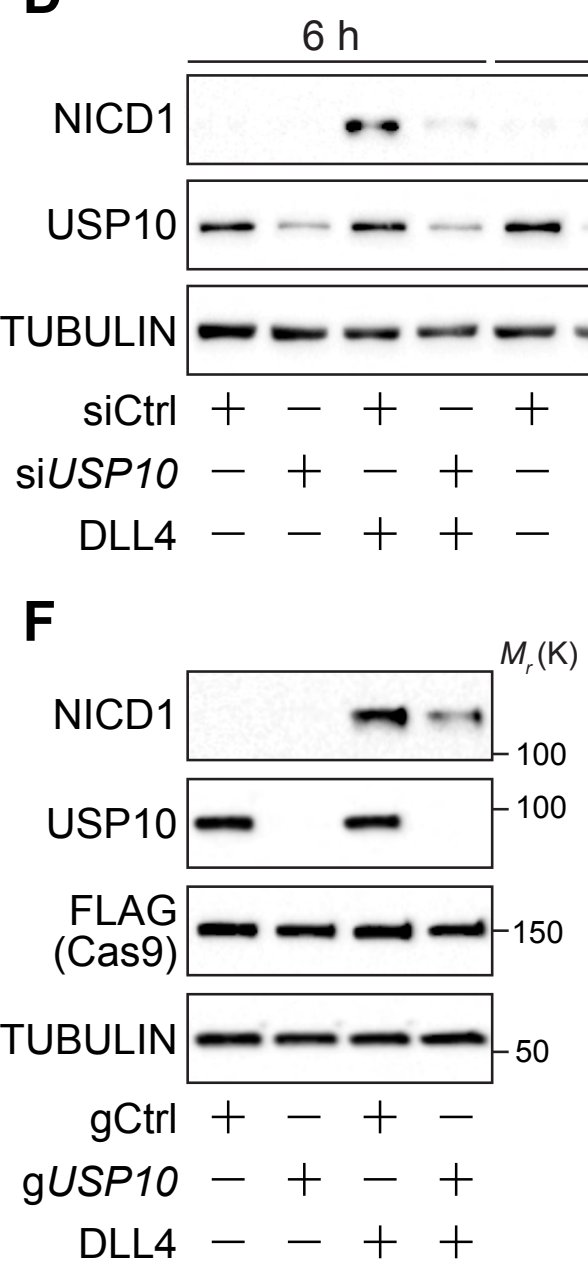

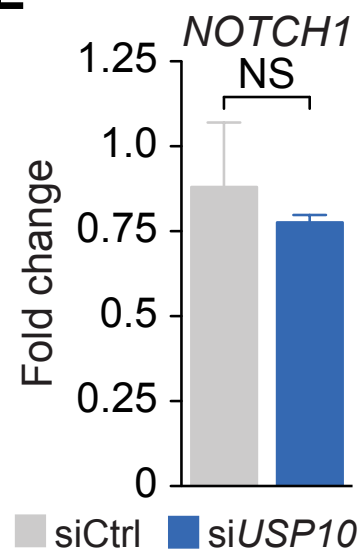

LentiUSP10 $10^{\mathrm{GFP}}-+-+$

DLL4 $-\quad++$

C

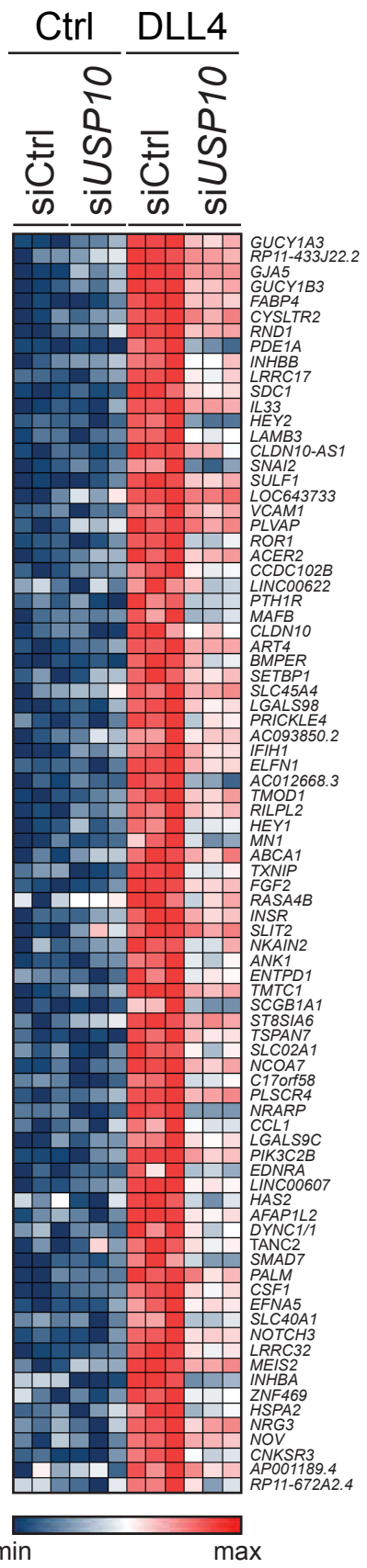


A

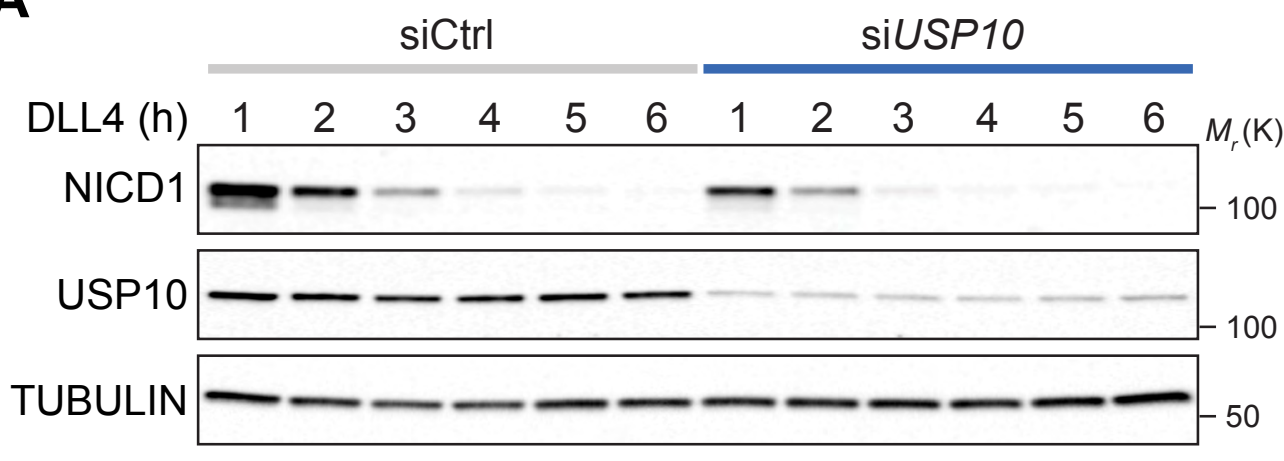

B
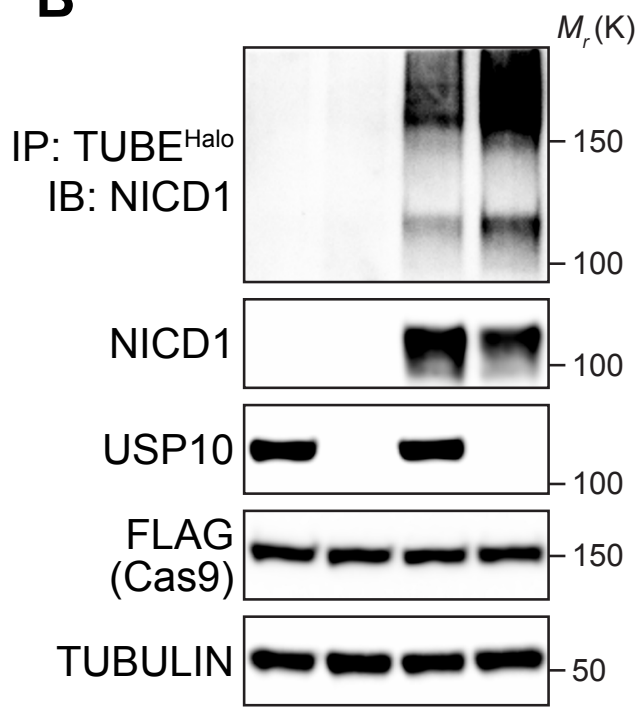

gCtrl +-+gUSP10 -+-+

DLL4 - -++
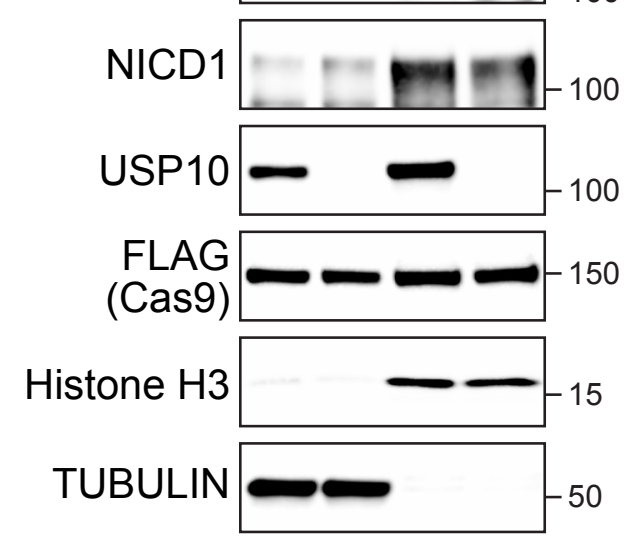
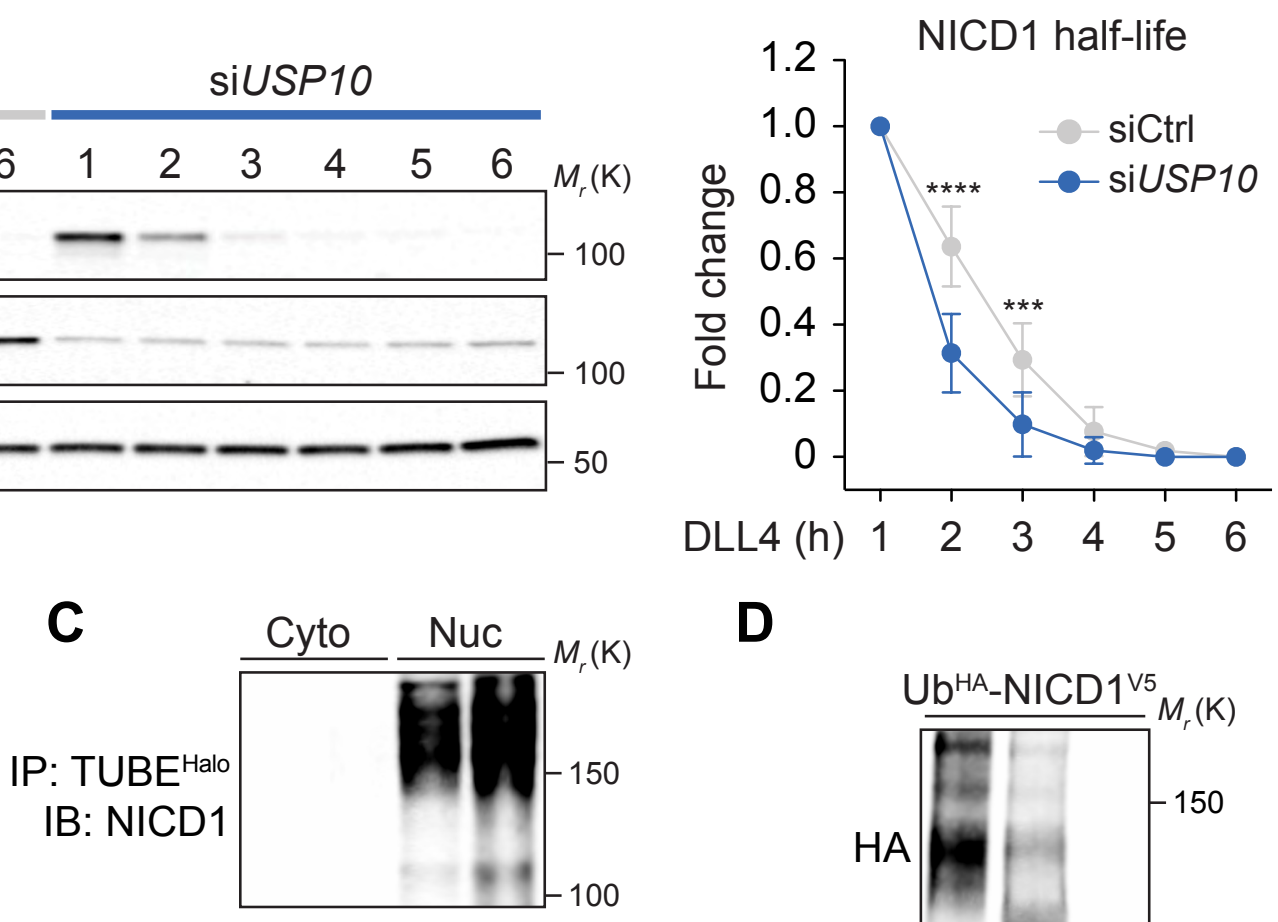

$$
\begin{aligned}
& \text { gCtrl }+-+- \\
& \text { gUSP10 }-+-+ \\
& \text { DLL4 }++++
\end{aligned}
$$

D

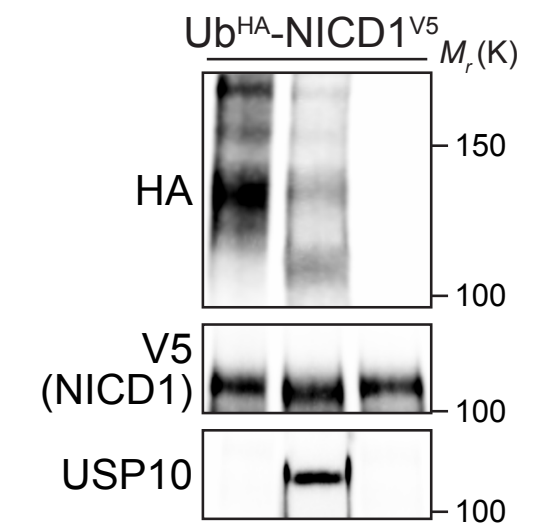

$$
\begin{aligned}
& \text { rUSP10 - + - } \\
& \text { rUSP2 cc }-\quad+
\end{aligned}
$$




\section{Figure 4}
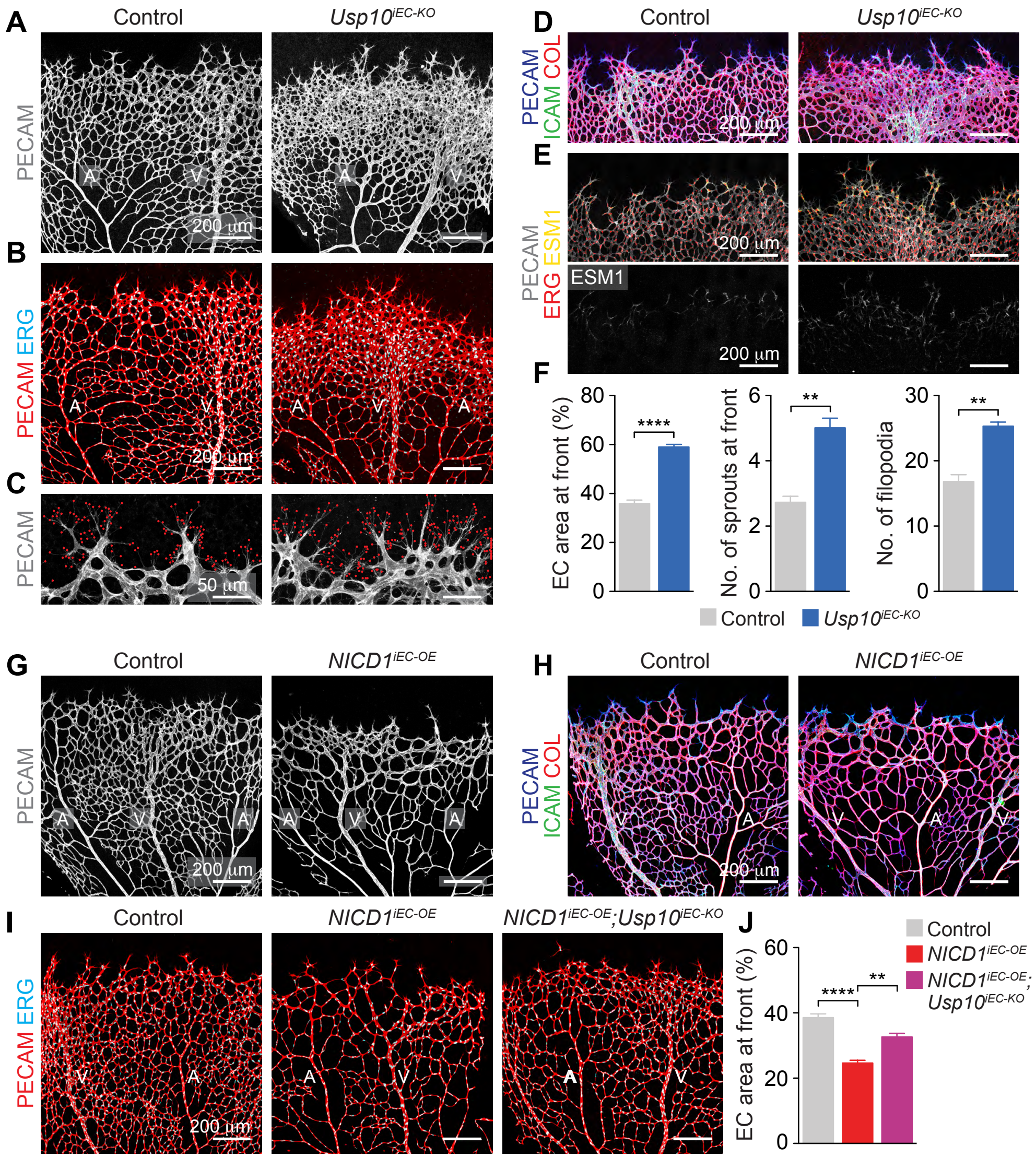
A

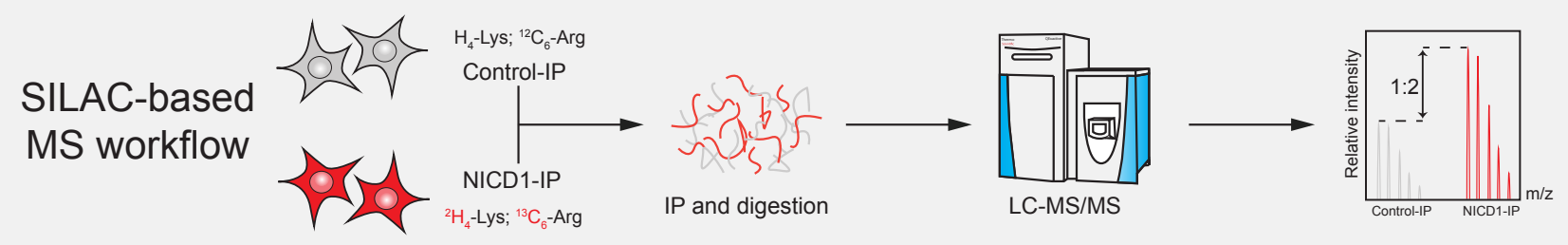

B

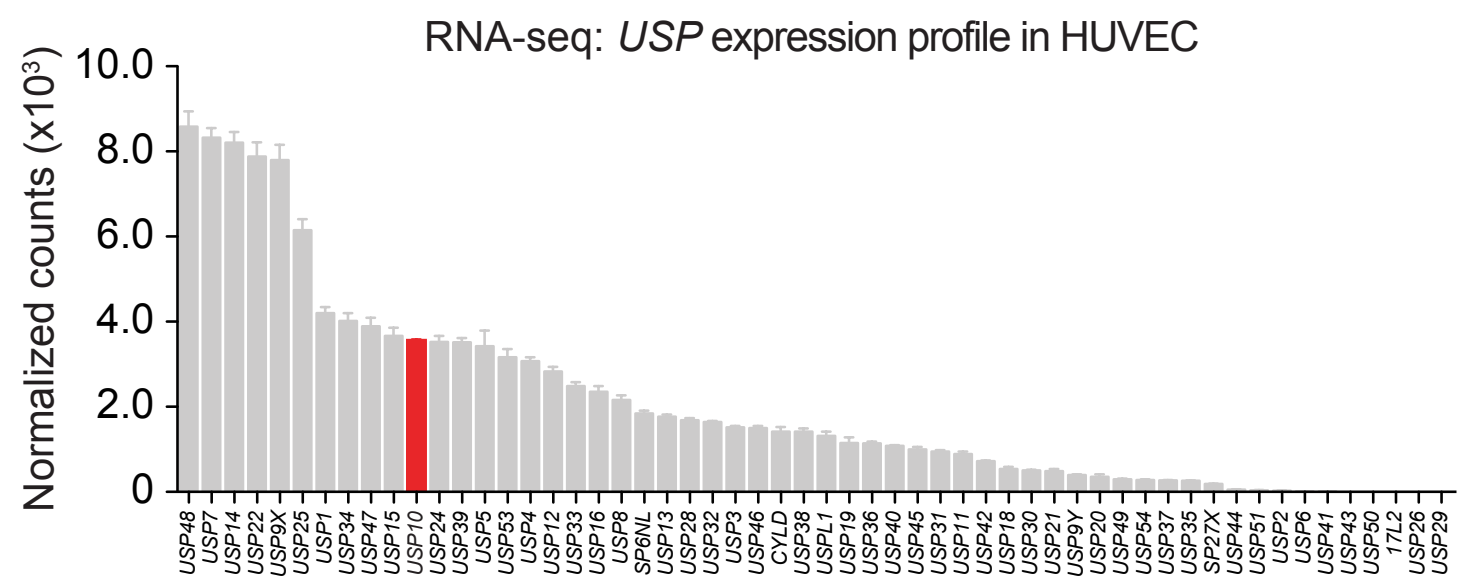

C

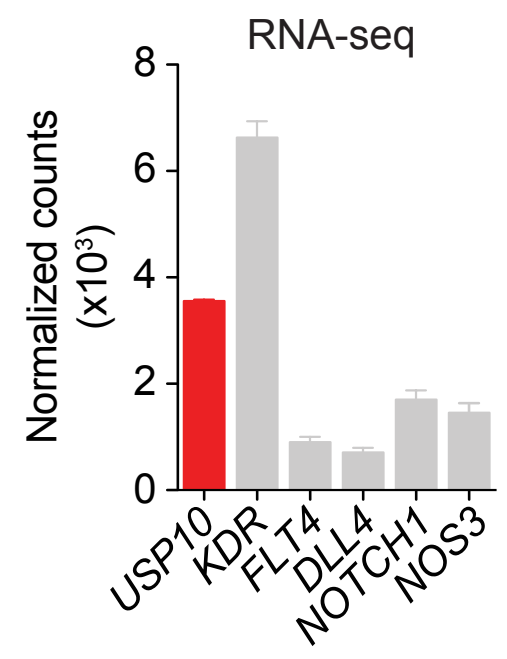

D

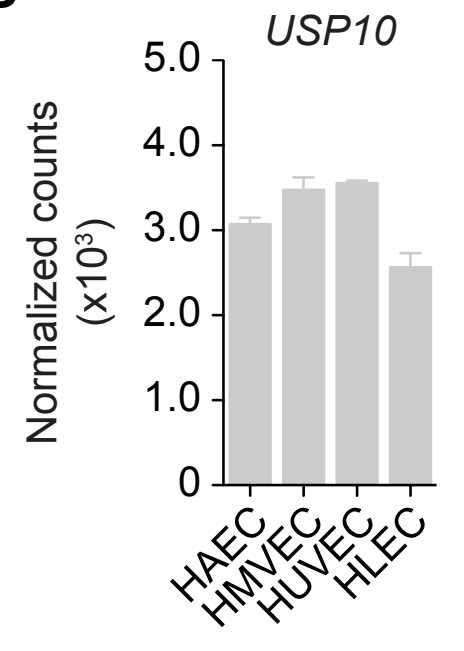

E

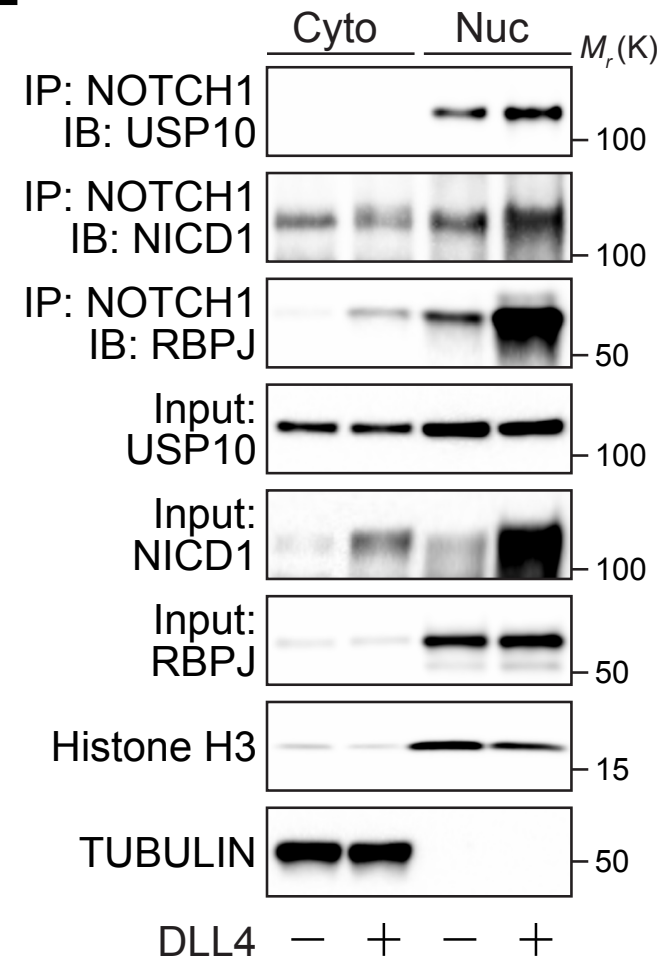


A

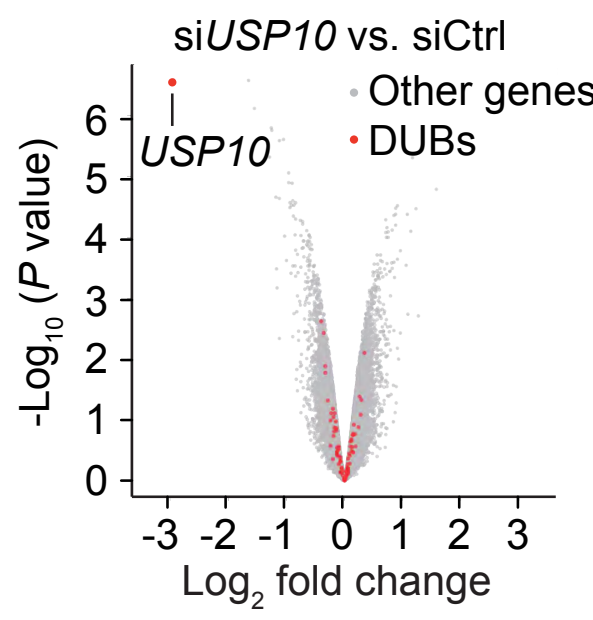

B

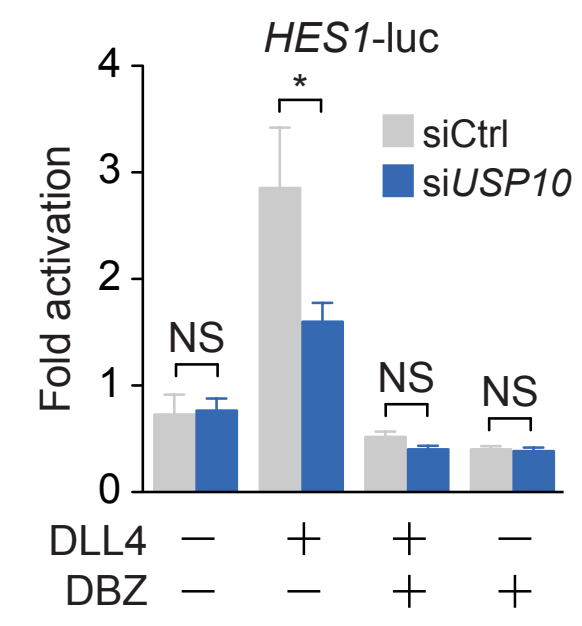

Ctrl DLL4

C $\equiv$ के $=\frac{5}{\text { के }}$

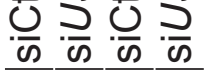

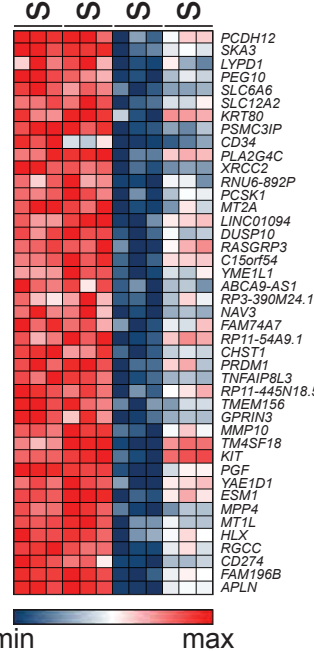

E
D

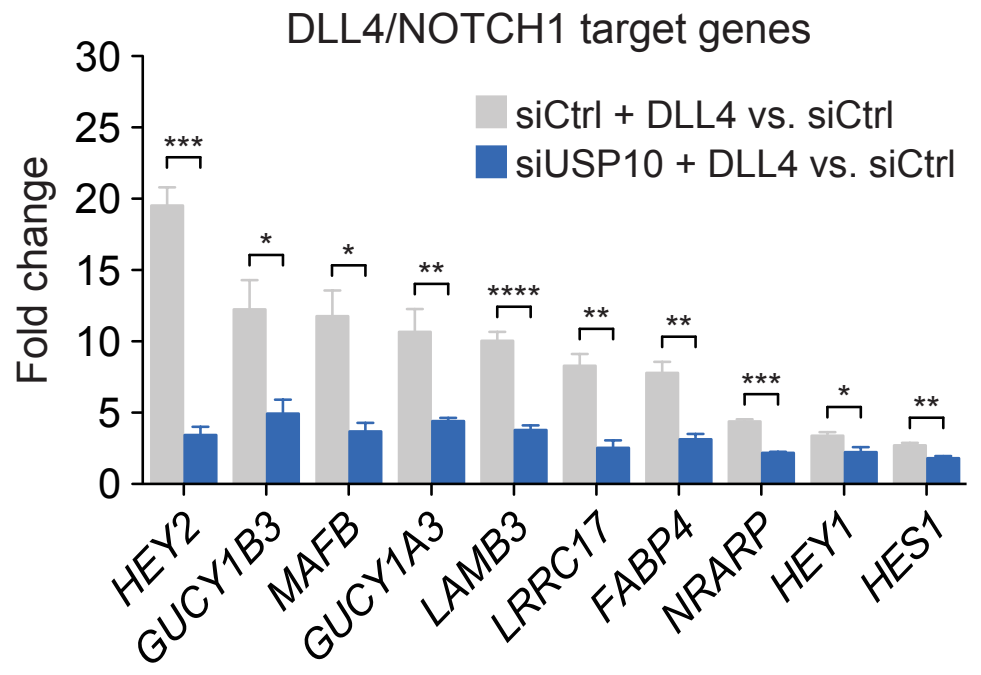

HEY2

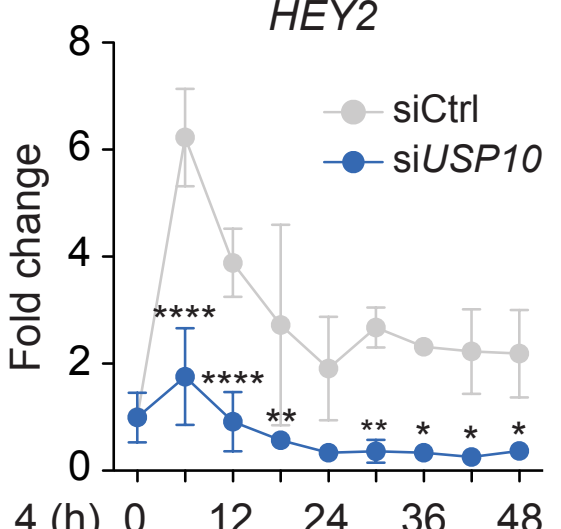

F

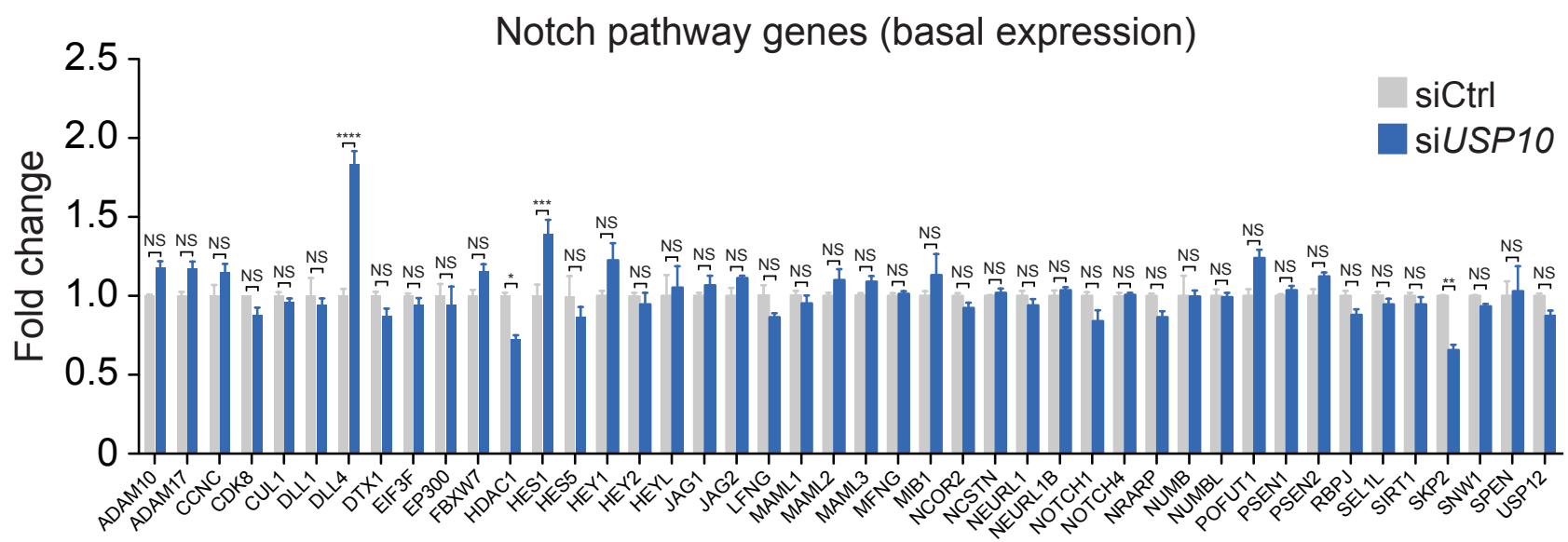




\section{Figure S3}

A B

siCtrl a siUSP10

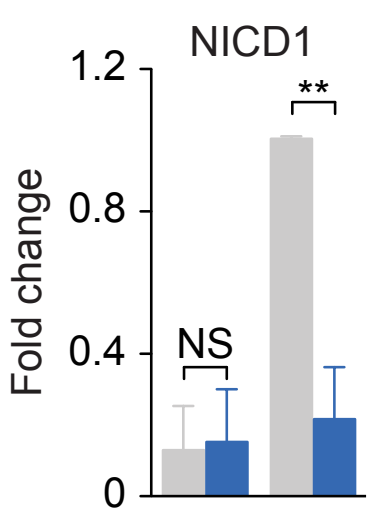

DLL4 - +

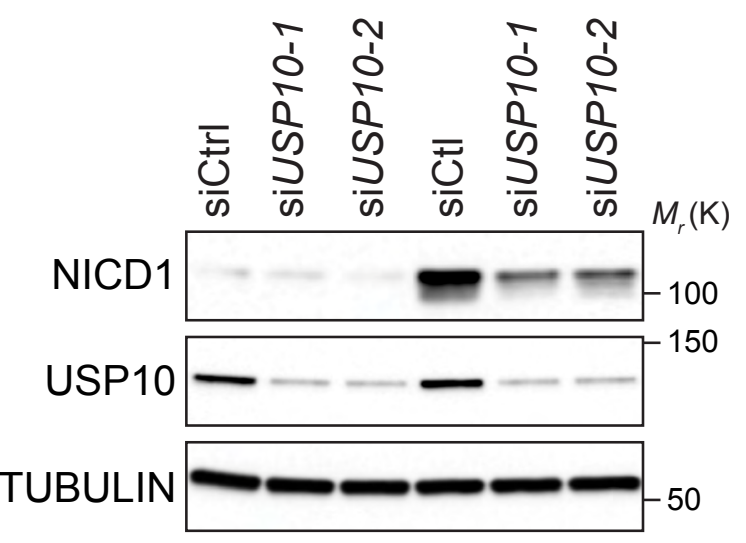

DLL4 $-\quad-\quad+++$
C

gCtrl a gUSP10

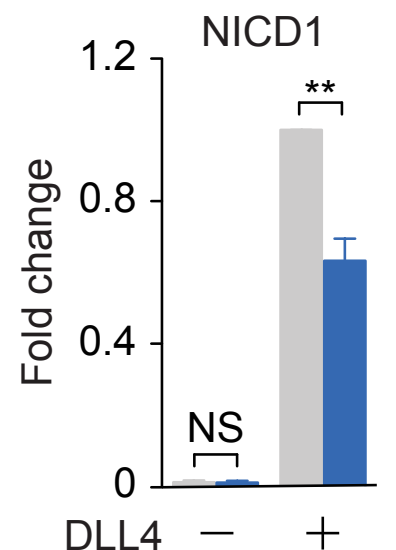

D

gCtrl gUSP10

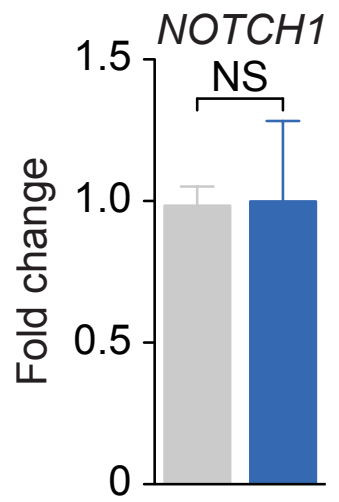

E

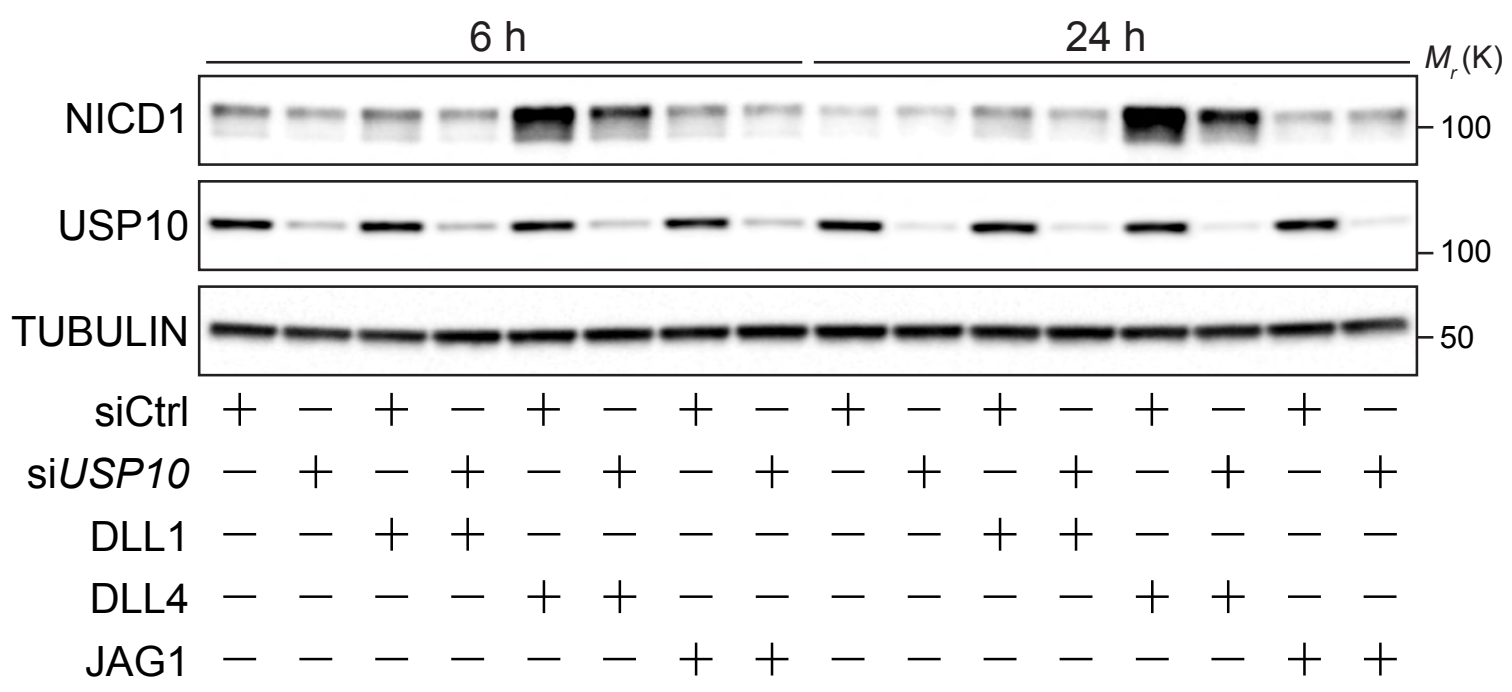

$\mathbf{F}$

G
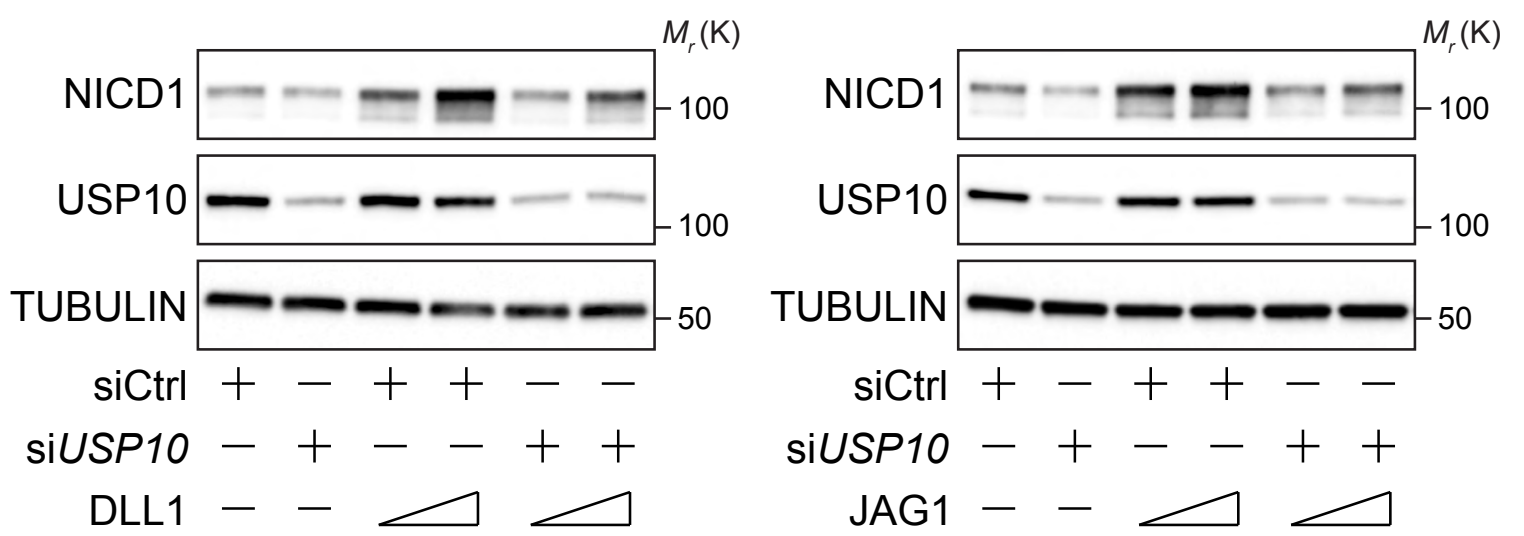


\section{Figure S4}

A
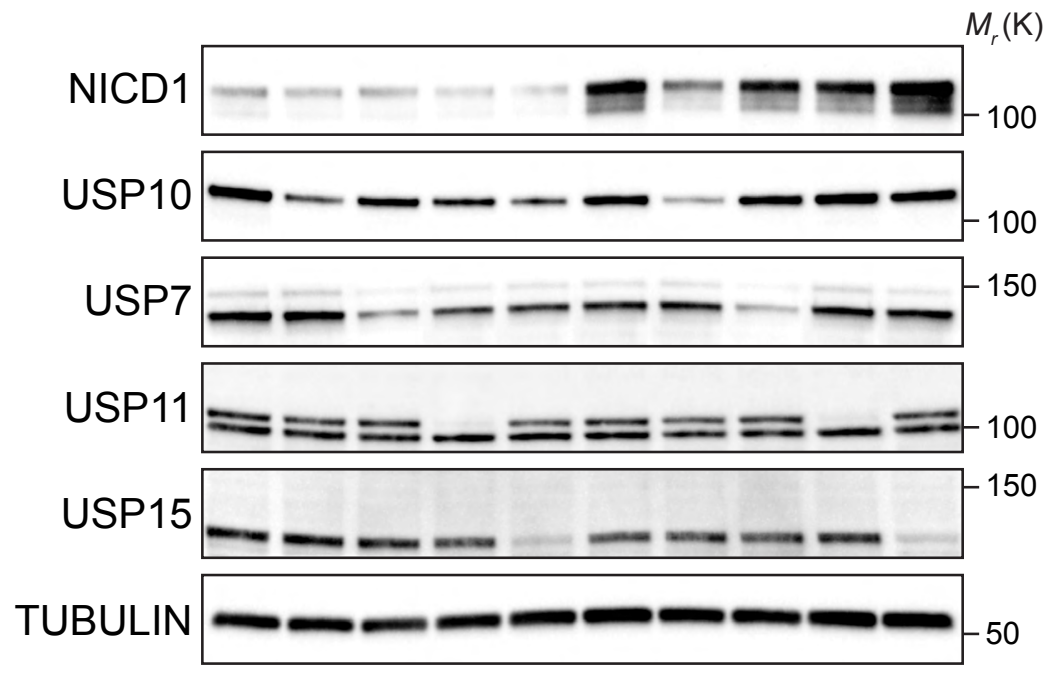

$$
\begin{array}{r}
\text { siCtrl }+----+---- \\
\text { siUSP10 }+----+--- \\
\text { SiUSP7 - + - - }-- \\
\text { siUSP11 - - + - - }- \\
\text { siUSP15 - - + - - }+ \\
\text { DLL4 - - - + + + + }
\end{array}
$$

B

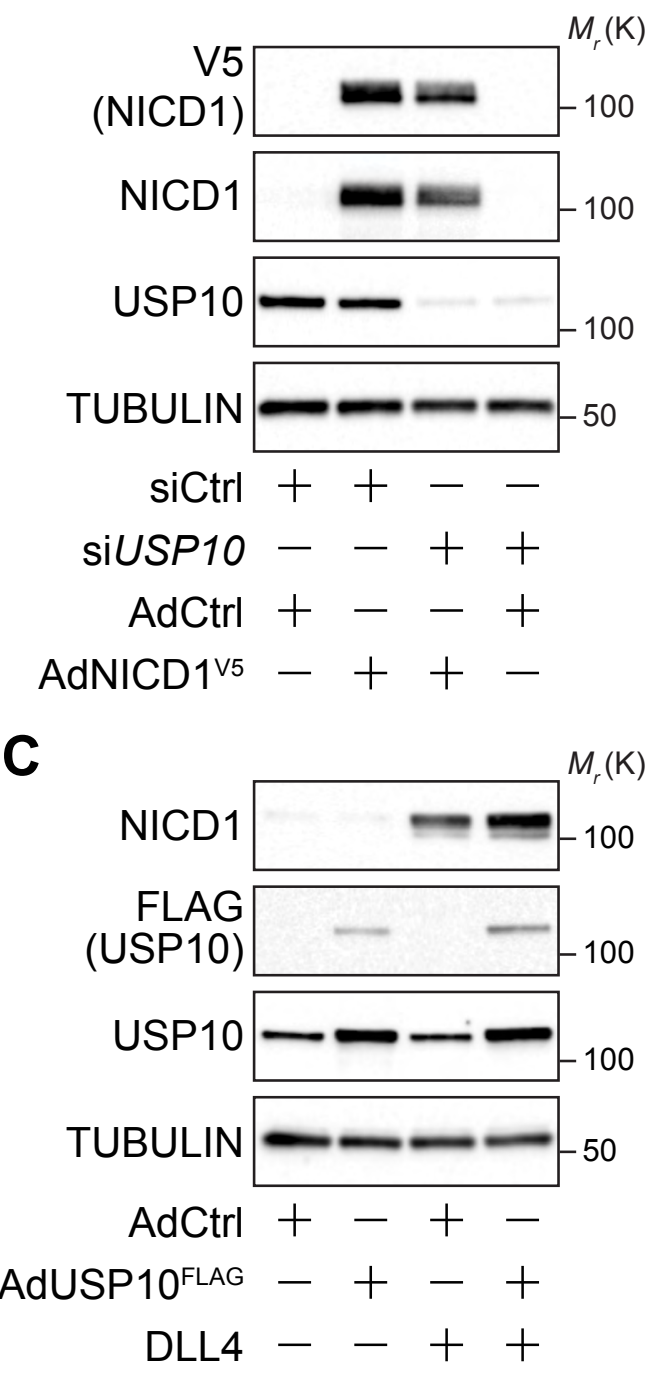




\section{Figure S5}

A B

B C
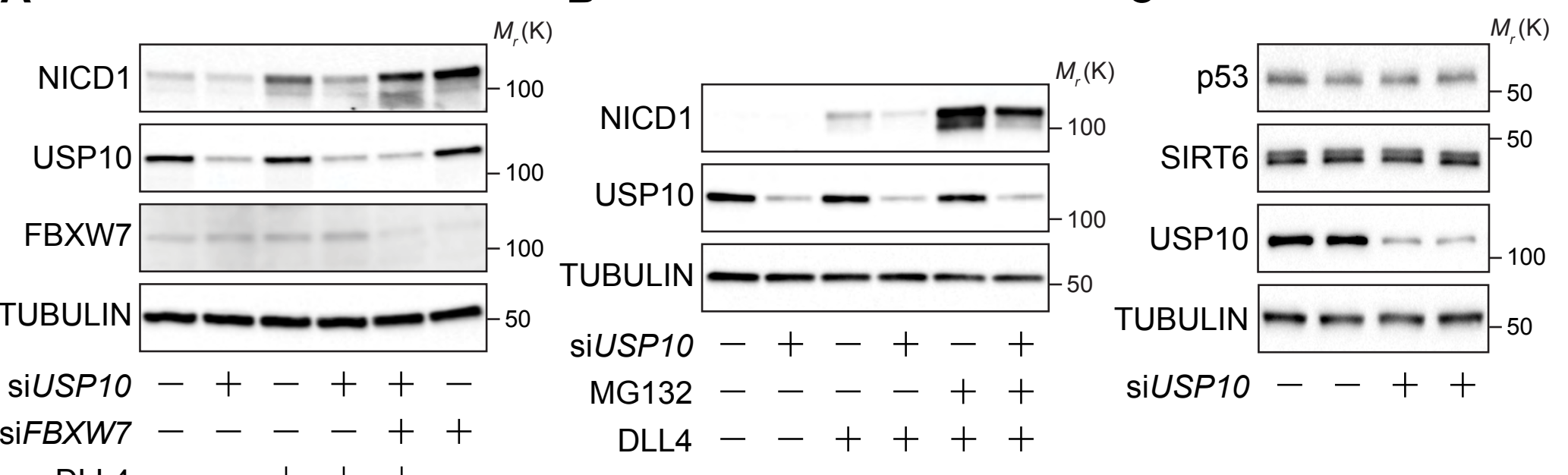

DLL4 - - + + -

D

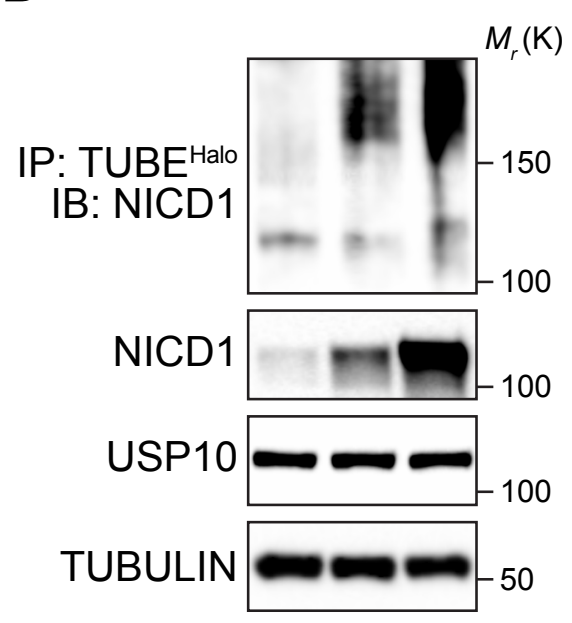

$$
\text { DLL4 - + + }
$$$$
\text { MG132 - - + }
$$

E

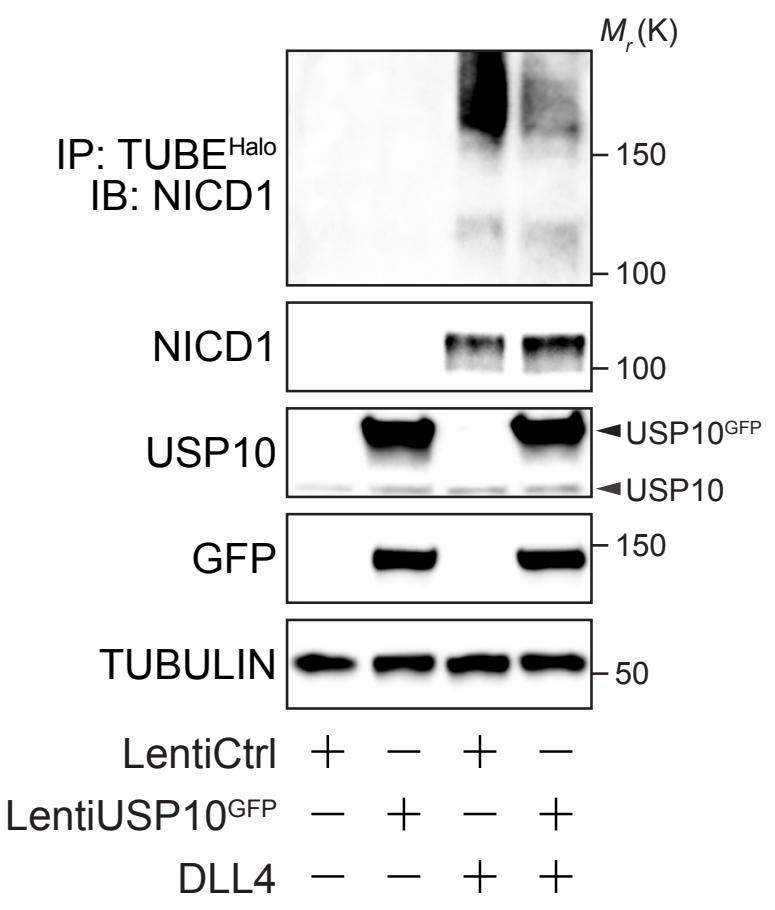

$\mathbf{F}$

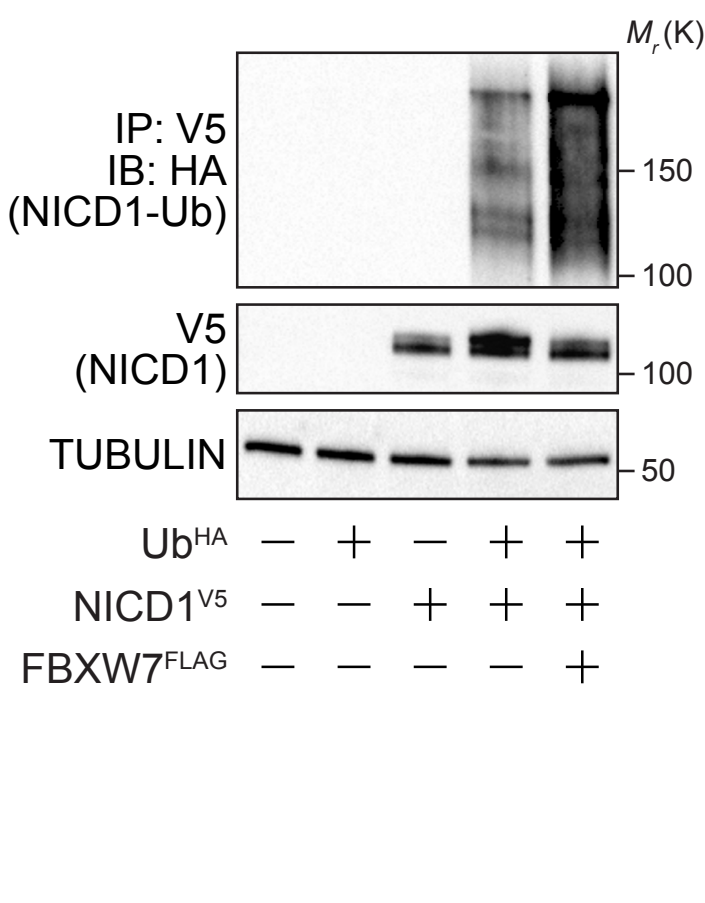




\section{Figure S6}

A

Targeting strategy

Wild-type Usp10 locus
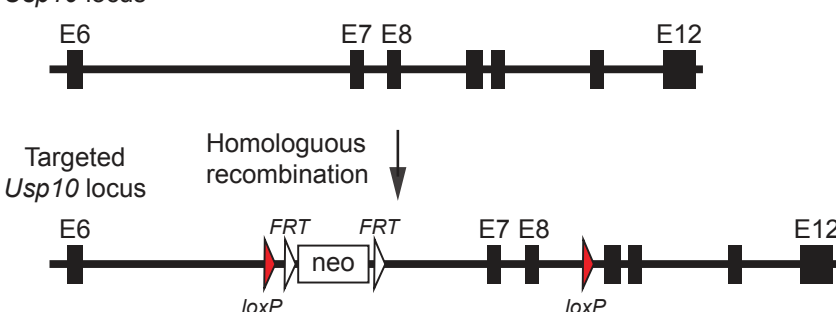

\begin{tabular}{|c|c|c|}
\hline Usp10 $10^{+/+}$ & Usp10/- & Usp10\%- \\
\hline $25(46.3 \%)$ & $29(53.7 \%)$ & $0(0 \%)$ \\
\hline
\end{tabular}

Postnatal genotypes
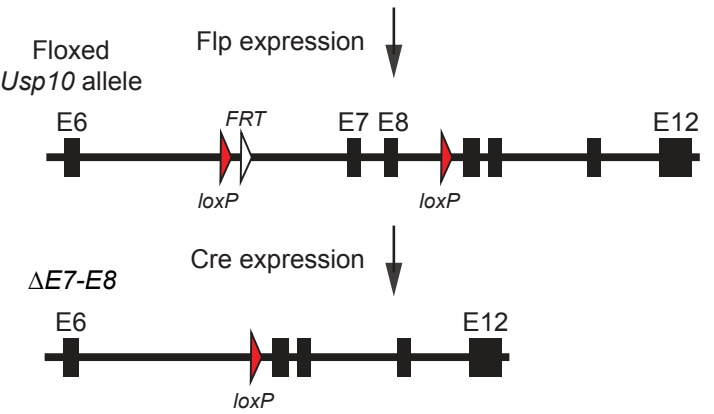

Usp10 $10^{+/} \times$Usp10 $10^{+/-}$intercross

C

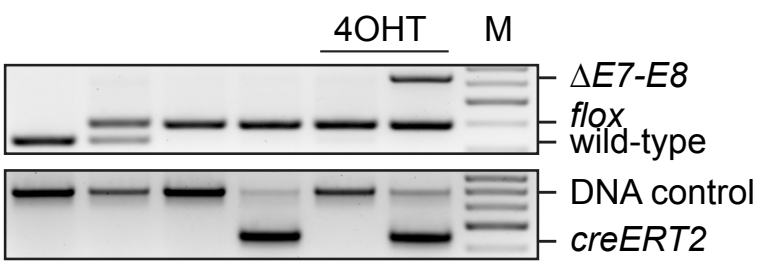

D
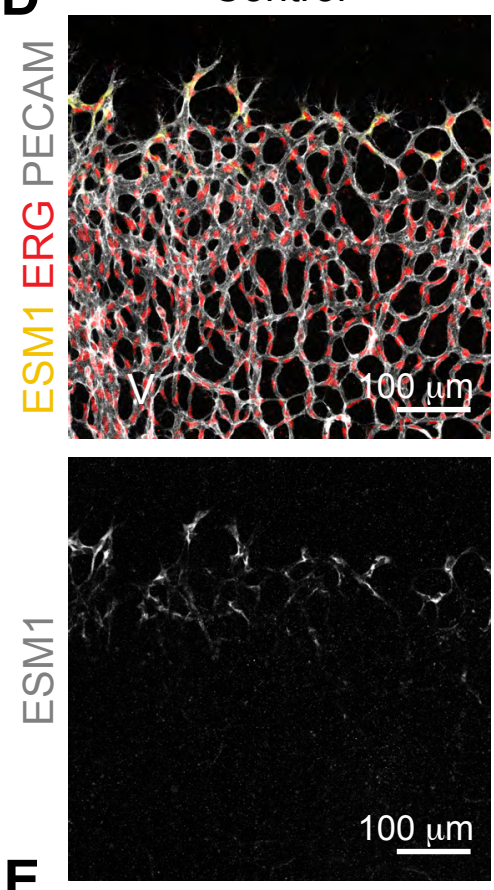

E

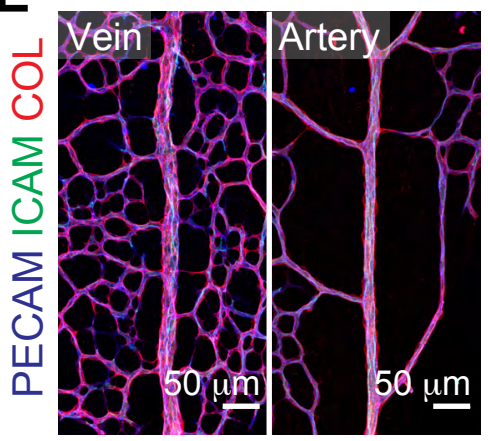

Usp10 ${ }^{\mathrm{iEC}-K O}$
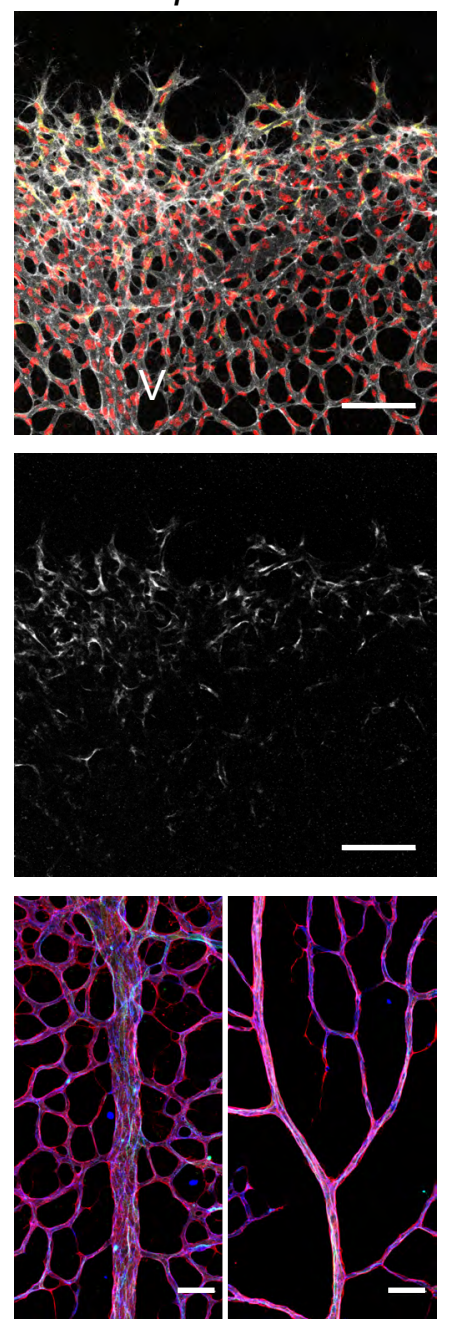

Control Usp10iEC-Ko
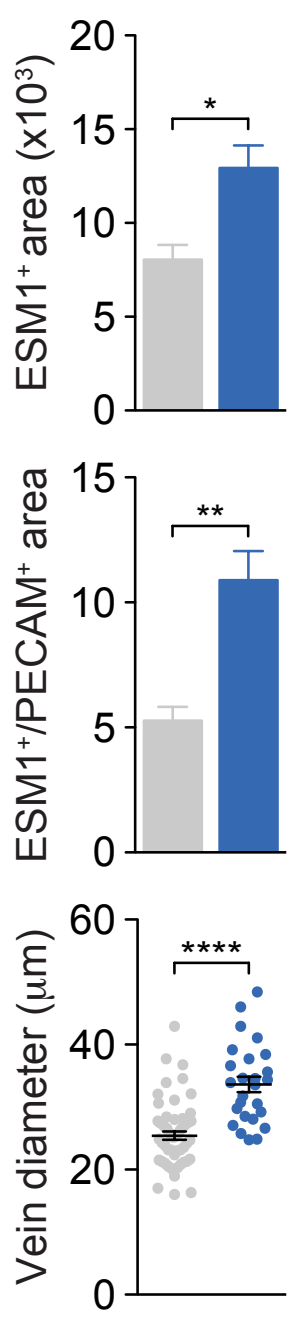


\section{Figure S7}

A

Cre-inducible NICD1overexpression allele
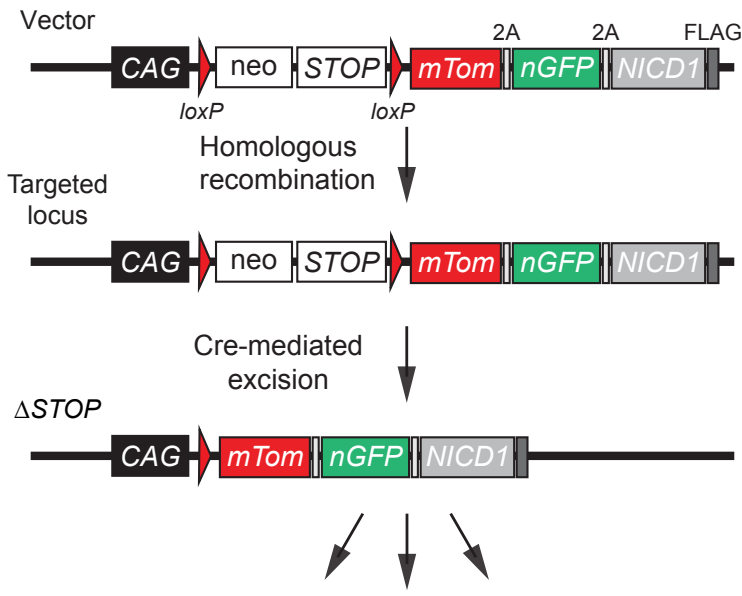

mTOMATO nGFP NICD1FLAG
B
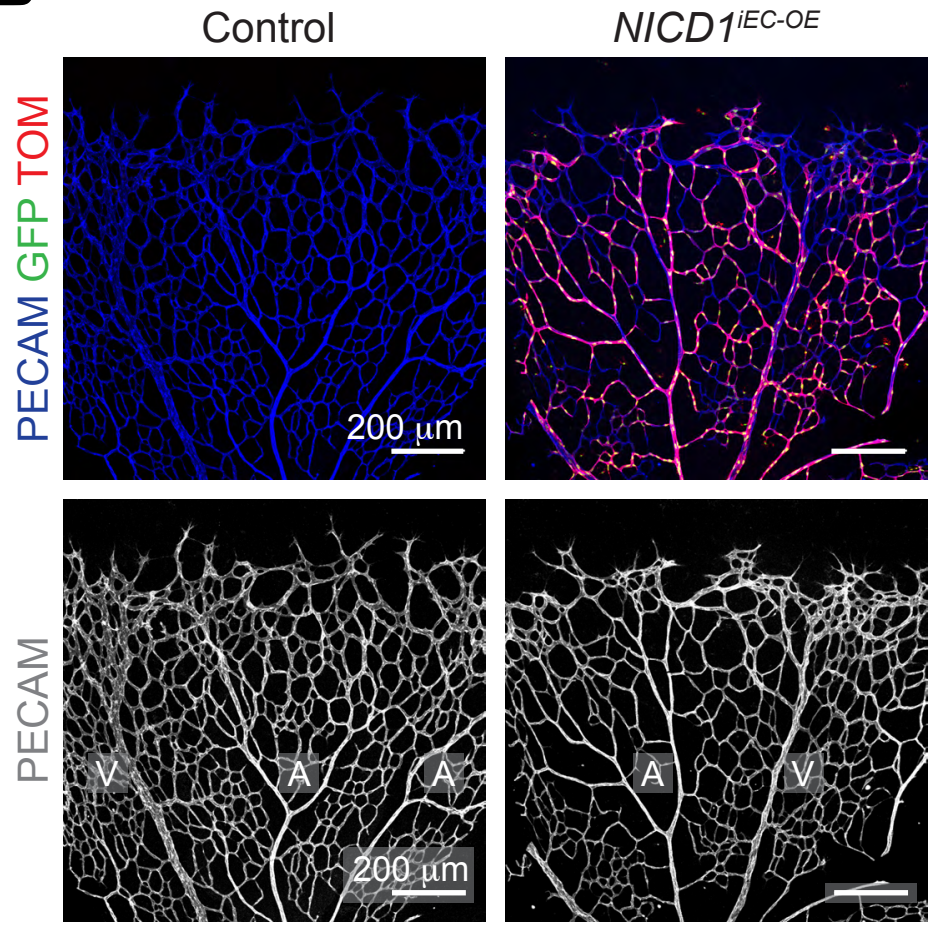

D

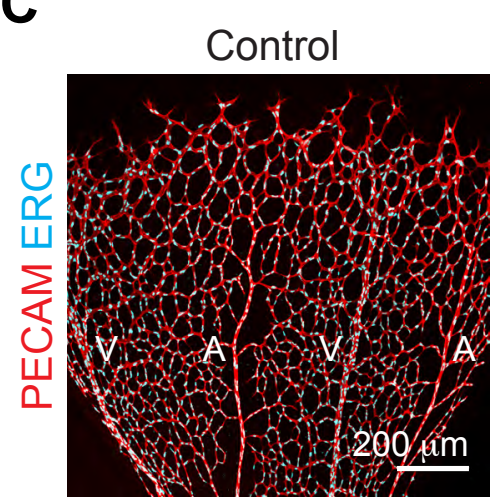

NICD $1^{\text {iEC-OE }}$

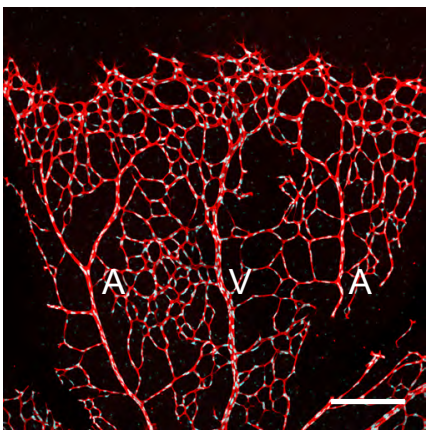

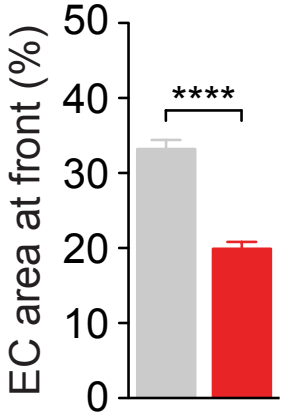

Control NICD1 $1^{\mathrm{iEC}-O E}$
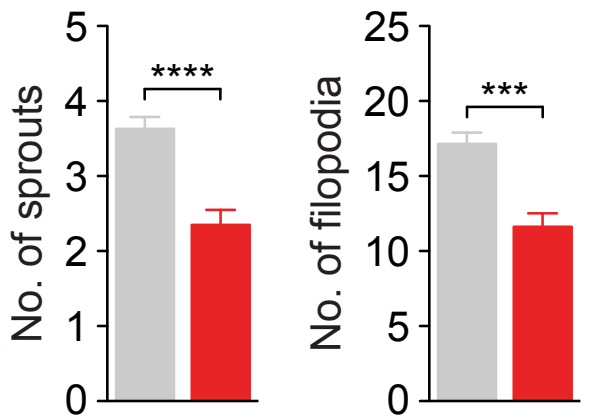


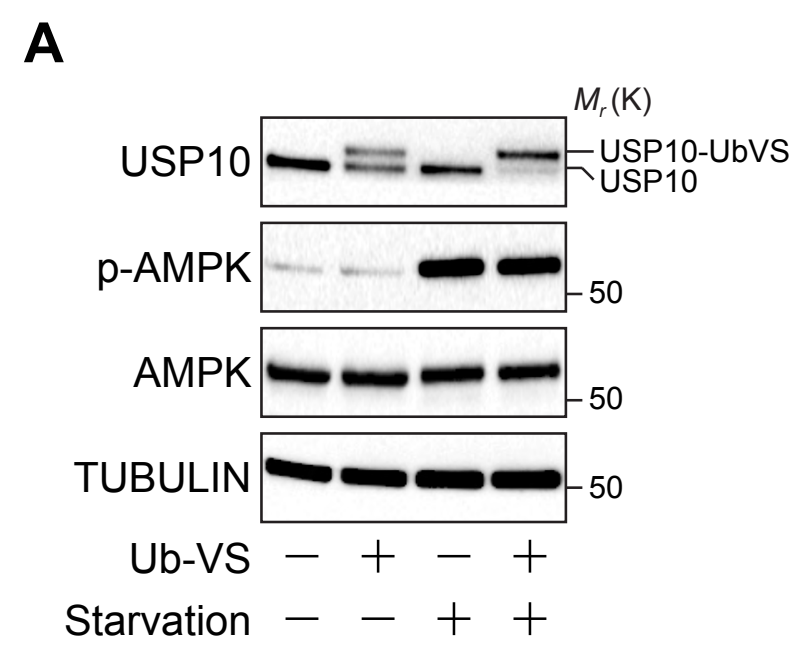

B

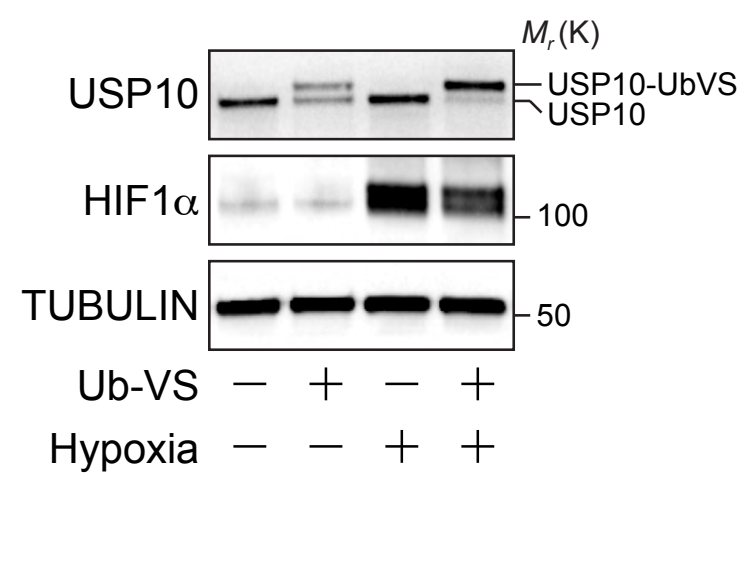




\title{
Science $\triangle$ IAAAS
}

\section{Supplementary Materials for}

\section{Deubiquitinase USP10 regulates Notch signaling in the endothelium}

\author{
R. Lim $^{1 \S}$, T. Sugino ${ }^{1 \S}$, H. Nolte ${ }^{2}$, J. Andrade ${ }^{1}$, B. Zimmermann ${ }^{1}$, C. Shi ${ }^{1}$, A. Doddaballapur ${ }^{1}$, Y.- \\ T. Ong ${ }^{1}$, K. Wilhelm ${ }^{1}$, J.W.D. Fasse ${ }^{1}$, A. Ernst ${ }^{3,4}$, M. Kaulich ${ }^{5}$, K. Husnjak ${ }^{6}$, T. Boettger ${ }^{7}$, S. \\ Guenther $^{7}$, T. Braun ${ }^{7}$, M. Krüger ${ }^{2}$, R. Benedito ${ }^{8}$, I. Dikic ${ }^{9}$, M. Potente ${ }^{1,10^{*}}$ \\ Correspondence to: michael.potente@mpi-bn.mpg.de
}

This PDF file includes:

Materials and Methods

Figs. S1 to S8 


\section{Materials and Methods}

\section{Cell culture}

Pooled human umbilical vein endothelial cells (HUVECs) were purchased from Lonza (\#CC2519) and cultured in endothelial basal medium (EBM, Lonza) supplemented with hydrocortisone $(1 \mu \mathrm{g} / \mathrm{ml})$, bovine brain extract $(12 \mu \mathrm{g} / \mathrm{ml})$, gentamicin $(50 \mu \mathrm{g} / \mathrm{ml})$, amphotericin B (50 ng/ml), epidermal growth factor $(10 \mathrm{ng} / \mathrm{ml})$, and $10 \%$ fetal bovine serum (FBS, Life Technologies) or endothelial growth medium (EGM-Plus, Lonza) supplemented with hydrocortisone, L-Glutamine, bovine brain extract, gentamicin/amphotericin-B, epidermal growth factor, heparin, and 10\% FBS. HUVECs were tested negative for mycoplasma and cultured until the fourth passage. Cells were maintained at $37^{\circ} \mathrm{C}$ in a humidified atmosphere with 5\% $\mathrm{CO}_{2}$. The other endothelial subtypes - pooled human aortic (\#CC-2535), microvascular (\#CC-2813) and lymphatic endothelial cells (\#CC-2812) - were also purchased from Lonza and cultured according to manufacturer's protocol. Human embryonic kidney cells (HEK293T) were from Life Technologies and cultured in DMEM supplemented with $10 \%$ fetal calf serum, penicillin (100 units $/ \mathrm{ml})$ and streptomycin $(100 \mu \mathrm{g} / \mathrm{ml})$. Cells were maintained at $37^{\circ} \mathrm{C}$ in a humidified atmosphere with $5 \% \mathrm{CO}_{2}$.

\section{DLL4 stimulation and in vitro cell treatments}

Lyophilised recombinant human DLL4 (R\&D Systems) was reconstituted at $100 \mu \mathrm{g} / \mathrm{ml}$ in sterile PBS containing $0.1 \%$ bovine serum albumin. For stimulation of cultured endothelial cells, DLL4 was immobilised by coating culture dishes with $500 \mathrm{ng} / \mathrm{ml}$ DLL4 in PBS for $1 \mathrm{~h}$ at room temperature or for at least $12 \mathrm{~h}$ at $4^{\circ} \mathrm{C}$. To inhibit Notch signalling (by $\gamma$-secretase inhibition), 
HUVECs were treated with $0.08 \mathrm{mM}$ DBZ ((S,S)-2-[2-(3,5-Difluorophenyl)acetylamino]-N-(5methyl-6-oxo-6,7-dihydro-5H-dibenzo[b,d] azepin-7-yl) propionamide). Control groups were treated with the respective vehicles. When HUVECs were co-stimulated with DLL4 and DBZ, cells were pre-treated with DBZ for $1 \mathrm{~h}$ before being replated on DLL4-coated dishes. For the cycloheximide (CHX) chase experiments, HUVECs were incubated with $50 \mu \mathrm{g} / \mathrm{ml}$ CHX. For proteasome inhibition, cells were treated with $20 \mu \mathrm{M}$ MG132 for $4 \mathrm{~h}$.

\section{Transfections and plasmids}

Transient transfections of HEK293T cells were carried out with Lipofectamine 2000 transfection reagent (Life Technologies) according to the manufacturer's instructions. Construction of the NICD1-V5 plasmid was previously described (1). The plasmid encoding for the 3xFLAG-tagged USP10 was from Zhenkun Lou (2). The FLAG-tagged human full-length FBXW7 was a kind gift from Nikita Popov (3). A pcDNA3.1-based plasmid expressing HA-tagged ubiquitin was from the Dikic laboratory. plentiCRISPR v2 plasmids with inserted gRNAs for human USP10 were from the Kaulich laboratory. For lentiviral expression of a GFP-tagged USP10, USP10 was first cloned by PCR into pDONR221 (Invitrogen) and then subcloned into pcDNA-DEST53 (Invitrogen) by Gateway recombination. GFP-USP10 fusion was further subcloned into pLVXTetOne-Puro (Clontech).

\section{Adenoviral transductions}

Sub-confluent HUVECs were infected with adenoviruses to overexpress NICD1, USP10, UbV.10 and UbCtrl. NICD1 and USP10-expressing adenoviruses (AdGFP/NICD1-V5 and 
AdGFP/USP10-FLAG) were custom made by Vector Biolabs. GFP-expressing adenovirus (Vector Biolabs) were used as control. HUVECs were incubated in EBM (Lonza) containing $0.1 \%$ BSA for $4 \mathrm{~h}$. Prior to infection, adenoviruses were incubated for 10 min with $8 \mu \mathrm{g} / \mathrm{ml}$ polybrene (Santa Cruz) to facilitate the infection. The mixture was then applied to the HUVECs cultured in EBM containing 0.1\% BSA and incubated for $4 \mathrm{~h}$. Thereafter, the cells were washed five times with Hank's Balanced Salt Solution (Life Technologies) and cultured in EBM with $10 \%$ FBS and supplements.

\section{Lentiviral transductions}

Virus production was performed by transfection of HEK293T cells with psPAX2, pMD2.G and transfer plasmids. Viruses were harvested 48 and 72 hours after transfection, and incubated with HUVECs for 8 hours in presence of $4 \mu \mathrm{g} / \mathrm{ml}$ polybrene (Santa Cruz). Fresh media containing 1 $\mu \mathrm{g} / \mathrm{ml}$ puromycin was added to select the transduced cells.

\section{RNA interference}

To silence USP10 gene expression, HUVECs were transfected with $50 \mathrm{nM}$ USP10 ONTARGET SMARTpool siRNA (Dharmacon), comprising a pool of four individual siRNA duplexes. As a control, a non-targeting siRNA pool was used (Dharmacon). HUVECs were grown to $70 \%$ confluency and transfected with Lipofectamine RNAiMAX (Life Technologies) according to the manufacturer's instructions. Experiments were recapitulated using two different siRNAs (Silencer Select siRNA, Ambion) To silence FBXW7, USP7, USP9X, USP11 or USP15 
expression, $50 \mathrm{nM}$ of the respective siRNA pool was used (ON-TARGET SMARTpool siRNA, Dharmacon).

\section{SILAC-based interactome studies}

For stable isotope labeling with amino acids in cell culture (SILAC) experiments, HEK293T cells were cultured for two weeks in growth medium (SILAC DMEM (\#E15-086, PAA), 10\% dialysed fetal calf serum, penicillin (100 units/ml), streptomycin $(100 \mu \mathrm{g} / \mathrm{ml})$, gentamycin (100 $\mu \mathrm{g} / \mathrm{ml}$ )) containing naturally occurring (Arg-0/Lys-0) amino acids, or the heavy isotopic variants Arg-6/Lys-4 (Silantes GmbH). The unlabeled ('light') cells were transfected with empty vector while the 'heavy' labelled cells were transfected with plasmid expressing NICD1-V5. At $24 \mathrm{~h}$ post-transfection, cells were lysed in IPLS buffer (50 mM Tris-HCL pH7.5, $120 \mathrm{mM} \mathrm{NaCl,} 0.5$ $\mathrm{mM}$ EDTA and 0,5\% Nonidet P-40) freshly supplemented with $1 \times$ EDTA-Free Complete Protease Inhibitor Cocktail (Roche) and $1 \mathrm{mM}$ phenylmethylsulfonyl fluoride. Samples were cleared by centrifugation and protein concentrations determined by the Bradford procedure. The labeled cell lysates were mixed in a 1:1 ratio. The samples were pre-cleared with A/G agarose beads (Santa Cruz) for $1 \mathrm{~h}$ at $4^{\circ} \mathrm{C}$ prior to immunoprecipitation (IP). Samples were incubated with anti-V5 agarose affinity gel (Sigma, \#A7345) overnight at $4^{\circ} \mathrm{C}$. Beads were then washed five times in lysis buffer and enzymatic digestion of the immunoprecipitated proteins was performed as previously described (4). Briefly, the immunoprecipitated proteins were eluted by boiling the beads in NuPAGE LDS Sample Buffer containing $50 \mathrm{mM}$ DTT at $70^{\circ} \mathrm{C}$ for $10 \mathrm{~min}$. The eluate was separated by one-dimensional SDS-polyacrylamide gel electrophoresis. Each gel lane was divided into seven parts and further diced into $1 \mathrm{~mm}^{2}$ cubes. The gel pieces were washed several times before the proteins were reduced $\left(10 \mathrm{mM}\right.$ DTT at $56{ }^{\circ} \mathrm{C}$ for $\left.30 \mathrm{~min}\right)$ and 
alkylated (55 mM IAA, $30 \mathrm{~min}$, room temperature in the dark). After a few more washes, $40 \mu \mathrm{l}$ of $12 \mathrm{ng} / \mu \mathrm{l}$ trypsin (Sigma) was added to the gel pieces and allowed to incubate on ice for 30 min. $50 \mathrm{mM}$ of ammonium bicarbonate buffer was then added to cover all gel pieces and keep them wet during enzymatic cleavage overnight at $37^{\circ} \mathrm{C}$. The generated peptides were extracted from the gel pieces using an increasing content of acetonitrile and concentrated in a speed vac. Prior to LC-MS/MS analysis, samples were primed using the STAGE tip technique (5).

\section{Mass spectrometric analysis}

Peptides were eluted from C18 tips with $0.1 \%$ formic acid in $80 \%$ acetonitrile (ACN), concentrated in a speed vac to complete dryness and resuspended in buffer $\mathrm{A}(0.1 \%$ formic acid). The Liquid Chromatography tandem mass spectrometry (LC-MS/MS) equipment comprises an EASY nLC 1000 coupled to the quadrupole based QExactive instrument (Thermo Scientific) via a nano-spray electroionisation source. Peptides were separated on an in-house packed $50 \mathrm{~cm}$ column $(1.9 \mu \mathrm{m} \mathrm{C18}$ beads, Dr. Maisch $\mathrm{GmbH})$ using a binary buffer system: (A) $0.1 \%$ formic acid and (B) $0.1 \%$ formic acid in $80 \%$ acetonitrile. The content of buffer B was raised from $6 \%$ to $38 \%$ within $40 \mathrm{~min}$ and followed by an increase to $63 \%$ within $5 \mathrm{~min}$. Then, within $5 \mathrm{~min}$ buffer B fraction was raised to $95 \%$ and held there for $5 \mathrm{~min}$ after which it was decreased to $5 \%$ within 2 min and held there for 3 min before the next sample was loaded on the column. MS1 spectra were acquired using a resolution of 70,000 (at $200 \mathrm{~m} / \mathrm{z}$ ), an Automatic Gain Control (AGC) target of 3e6, and a maximum injection time of $20 \mathrm{~ms}$ in a scan range of 300-1750 Th. Using a data dependent mode, the 10 most intense peaks were selected for isolation and fragmentation in the HCD cell using a normalized collision energy of 25. Dynamic exclusion was enabled and set to $30 \mathrm{~s}$. The MS/MS scan properties were: 35,000 resolution at $200 \mathrm{~m} / \mathrm{z}$, an 
AGC target of 5e5, an isolation window of $1.8 \mathrm{Th}$ and a maximum injection time of $120 \mathrm{~ms}$. Raw data were processed using MaxQuant 1.5.1.2 (6) and the implemented Andromeda search engine (7). MS/MS spectra were correlated against the human Universal Protein Resource (UniProt) database including a list of common contaminants. We used $7 \mathrm{ppm}$ and $4.5 \mathrm{ppm}$ MS/MS tolerances for first and main search respectively. The FDR at the peptide-spectrummatch and the protein level was controlled by the implemented decoy algorithm using the revert database to $1 \%$. "Match-between runs" and "Re-quantify" algorithms were activated and used by default settings. N-term acetylation and oxidation at methionine residues were defined as variable modifications, whereas carbamidomethylation at cysteine residues was defined as a fixed modification. $\log _{2}$ SILAC ratios were normalised prior to further processing by median subtraction.

\section{Immunoprecipitations}

For immunoprecipitations of overexpressed proteins, cells were lysed in IPLS buffer (50 mM Tris-HCL pH7.5, $120 \mathrm{mM} \mathrm{NaCl}, 0.5 \mathrm{mM}$ EDTA and 0.5\% Nonidet P-40) freshly supplemented with $1 \times$ EDTA-Free Complete Protease Inhibitor Cocktail (Roche) and $1 \mathrm{mM}$ phenylmethylsulfonyl fluoride for $30 \mathrm{~min}$ on ice. Samples were cleared by centrifugation and protein concentrations determined by the Bradford procedure. Equal amounts of proteins were used for the immunoprecipitation (IP) and 5\% from the total lysates were used for input controls. The total lysates were subjected to a 30-min pre-clearing with A/G agarose beads (Santa Cruz) with gentle rotation at $4^{\circ} \mathrm{C}$ before the IP step. Epitope tag immunoprecipitations were performed using anti-V5 agarose affinity gel (Sigma, \#A7345) or anti-FLAG M2 affinity gel (Sigma, \#F2426) at $4^{\circ} \mathrm{C}$ with gentle rotation overnight. For immunoprecipitation for endogenous NICD1, 
HUVECs were lysed in IPLS buffer (50 mM Tris-HCL pH7.5, $120 \mathrm{mM} \mathrm{NaCl}, 0.5 \mathrm{mM}$ EDTA and $0.5 \%$ Nonidet P-40) freshly supplemented with $1 \times$ EDTA-Free Complete Protease Inhibitor Cocktail (Roche) and $1 \mathrm{mM}$ phenylmethylsulfonyl fluoride for $60 \mathrm{~min}$ on ice. Samples were cleared by centrifugation and protein concentrations determined by the Bradford procedure. Immunoprecipitation for endogenous NICD1 was performed using anti-NOTCH1 (Cell Signaling Technology, \#3447, 1:50) at $4^{\circ} \mathrm{C}$ with gentle rotation 1-h incubation followed by a 1-h incubation with Protein G Sepharose 4 Fast Flow beads (GE Healthcare). The immunoprecipitates were washed three times with lysis buffer containing $500 \mathrm{mM} \mathrm{NaCl}$ and once with $10 \mathrm{mM}$ Tris-HCl, pH8.0, and then eluted by boiling the beads in SDS sample buffer at $95^{\circ} \mathrm{C}$ for $5 \mathrm{~min}$.

\section{Cell Fractionation}

Cytoplasmic and nuclear fractions were separated by using NE-PER Nuclear and Cytoplasmic Extraction Reagents (Thermo Scientific).

\section{Western blot analysis and antibodies}

Western blot analyses were performed with precast gradient gels (Bio-Rad) using standard methods. Briefly, cells were lysed in RIPA buffer (Sigma; $150 \mathrm{mM} \mathrm{NaCl}, 1.0 \%$ IGEPAL CA$630,0.5 \%$ sodium deoxycholate, $0.1 \%$ SDS, and $50 \mathrm{mM}$ Tris, $\mathrm{pH} 8.0)$ supplemented with $1 \times$ EDTA-Free Complete Protease Inhibitor Cocktail (Roche) and $1 \mathrm{mM}$ phenylmethylsulfonyl fluoride. Proteins were separated by SDS-PAGE (Tris-glycine gels with Tris/glycine/SDS buffer, Bio-Rad) and transferred onto nitrocellulose membranes using the Trans Turbo Blot system 
(Bio-Rad). Membranes were probed with specific primary antibodies and then with peroxidaseconjugated secondary antibodies. The following primary antibodies were used: AMPK $\alpha$ (Cell

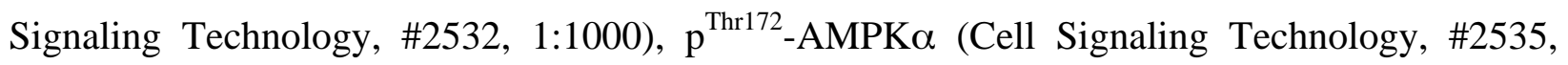
1:1000), Flag M2 (Sigma, \#F-3165, 1:1000), HA (Covance, clone 16B12, MMS-101P, 1:1000), HIF1 $\alpha$ (Cayman, \#10006421, 1:500), MAML1 (Cell Signaling Technology, \#12166, 1:1000), NOTCH1 (Cell Signaling Technology, \#3447, 1:1000), NICD1 (Cell Signaling Technology, \#2421, 1:1000), NICD1 (Cell Signaling Technology, \#4147, 1:1000), NICD1 (Abcam, \#Ab8925, 1:500), p53 (Cell Signaling Technology, \#9282, 1:1000), RBPSUH (D10A4) XP Rabbit mAb (Cell Signaling Technology, \#5313, 1:1000), SIRT6 (Cell Signaling Technology, \#2590, 1:1000), Tubulin (Cell Signaling Technology, \#2148, 1:5000), USP7 (Bethyl Laboratories, \#A300-033A, 1:1000), USP10 (Cell Signaling Technology, \#5553, 1:1000), USP11 (Bethyl Laboratories, \#A301-613A, 1:1000), USP15 (Bethyl Laboratories, \#A300-923A, 1:1000), V5 (ThermoFisher Scientific, \#R960-25, 1:5000), VEGFR2 (Cell Signaling Technology, \#2479, 1:1000) and $\mathrm{p}^{\text {Tyr1175}}$-VEGFR2 (Cell Signaling Technology, \#2478, 1:1000). Secondary antibodies are peroxidase-conjugated Goat IgGs (1:5000) purchased from Jackson Immuno Research Labs. The target proteins were visualised by chemiluminescence using an ECL detection kit (Clarity Western ECL Substrate, Bio-Rad) and a ChemiDoc MP Imaging System (Bio-Rad). Quantification of band intensities by densitometry was carried out using the Image Lab software (Bio-Rad).

\section{Luciferase assays}

Reporter assays in HUVECs were performed with the Dual-Luciferase Reporter Assay System (Promega) and Mithras LB 940 Reader (Berthold Technologies). The Notch-regulated luciferase 
reporter genes TP1, HES1 and 4xRBPJ were gifts from Ursula Zimber-Strobl, Mirko Schmidt and Diane Hayward, respectively. Plasmid encoding the Renilla luciferase reporter (pGL4.74hRluc/TK) was purchased from Promega. HUVECs were grown in $60 \mathrm{~mm}$ dishes to 60 - 70\% confluence and then transfected with $6 \mu \mathrm{g}$ of plasmids using the Trans Pass V reagents (New England Biolabs). HUVEC transfections were carried out according to the manufacturer's instructions. $24 \mathrm{~h}$ after co-transfection with the Notch luciferase reporters and the constitutive Renilla luciferase reporter, HUVECs were lysed and reporter assays performed according to the manufacturer's instructions. For siRNA experiments, cells were first transfected with siRNAs using Lipofectamine RNAiMAX (Life Technologies). After $24 \mathrm{~h}$, cells were then transfected with the Notch luciferase reporters and the constitutive Renilla luciferase reporter using the Trans Pass V reagents (New England Biolabs). For experiments in which Notch activity was induced by DLL4, transfected HUVECs were replated on DLL4-coated dishes $6 \mathrm{~h}$ after plasmid transfections. Luciferase activity was measured after an additional $24 \mathrm{~h}$. For treatment with the $\gamma$ secretase inhibitor DBZ, transfected HUVECs were pre-treated with DBZ for $1 \mathrm{~h}$ prior to the start of the 24-h DLL4 stimulation and DBZ treatment. Reporter activity was adjusted for the internal Renilla luciferase control and is expressed relative to control.

\section{Genome-wide transcriptomic and gene set enrichment analysis}

Total RNA quality was verified using the Agilent Bioanalyser and the 6000 Nano kit. RNA was labeled according to the Affymetrix Whole Transcript Sense Target Labeling protocol. Affymetrix GeneChip ${ }^{\circledR}$ Human Gene 2.0 ST arrays were hybridised, processed and scanned using the appropriate Affymetrix protocols. Data were analyzed using the Affymetrix expression console using the RMA algorithm, statistical analysis was done using DNAStar Arraystar. For 
gene set enrichment analysis (GSEA), gene set collections from the Molecular Signatures Database (MSigDB) 4.0 (http://www.broadinstitute.org/gsea/msigdb/) were used for the analysis of the endothelial NOTCH1 transcriptome.

\section{RNA-sequencing analysis}

For RNA-seq, RNA was isolated from HUVEC cells using the miRNeasy micro Kit (Qiagen) combined with on-column DNase digestion (DNase-Free DNase Set, Qiagen) to avoid contamination by genomic DNA. RNA and library preparation integrity were verified with a BioAnalyzer 2100 (Agilent) or LabChip Gx Touch 24 (Perkin Elmer). $4 \mu \mathrm{g}$ of total RNA was used as input for Truseq Stranded mRNA Library preparation following the low sample protocol (Illumina). Sequencing was performed on the NextSeq500 instrument (Illumina) using v2 chemistry, resulting in minimum of $37 \mathrm{M}$ reads per library with $1 \mathrm{x} 75 \mathrm{bp}$ single end setup. Processing of raw reads was performed as described (8).

\section{Quantitative real-time PCR (qRT-PCR) analysis}

RNA from HUVECs was isolated with the RNeasy Kit (Qiagen) according to the manufacturer's protocol. For cDNA synthesis, $2 \mu \mathrm{g}$ of total RNA was reverse transcribed by using M-MLV reverse transcriptase (Life Technologies). TaqMan Gene Expression Assays for HEY2 (Hs00232622_m1), NRARP (Hs01104102_m1), NOTCH1 (Hs01062014_m1), USP10 (Hs 00382490_m1) and $\beta$-ACTIN (Hs99999903_m1) were obtained from Applied Biosystems, and qPCR was carried out using the StepOnePlus real-time PCR system (Applied Biosystems). Gene 
expression was normalised to the endogenous control ( $\beta$-ACTIN) and calculated using the $\Delta \Delta \mathrm{Ct}$ method.

\section{Preparation of Halo-tagged TUBEs and pull-down assays}

TUBEs, tandem- repeated ubiquitin-binding entities $(9,10)$ were expressed in E. coli as Halotagged TUBEs. Cells were resuspended in lysis buffer containing $50 \mathrm{mM}$ Tris $\cdot \mathrm{HCl}, 150 \mathrm{mM}$ $\mathrm{NaCl}, 1 \mathrm{mM}$ EGTA, $1 \mathrm{mM}$ EDTA, 0.1\% (vol/vol) 2-mercaptoethanol, $1 \mathrm{mM}$ benzamidine and $0.2 \mathrm{mM}$ PMSF and lysed by sonication. After centrifugation, the supernatant was coupled to the

HaloLink resin (Promega) by incubation for $5 \mathrm{~h}$ at $4{ }^{\circ} \mathrm{C}$. The resin was washed extensively with $50 \mathrm{mM}$ Tris $\cdot \mathrm{HCl}$, pH 7.5, 0.5 M NaCl, 0.1 mM EDTA, $270 \mathrm{mM}$ sucrose, $0.03 \%$ (wt/vol) Brij 35, 0.1\% (vol/vol) 2-mercaptoethanol, $0.2 \mathrm{mM}$ PMSF, and $1 \mathrm{mM}$ benzamidine and stored at $4{ }^{\circ} \mathrm{C}$. After stimulation with DLL4 for $24 \mathrm{~h}$, HUVECs were extracted in lysis buffer containing $50 \mathrm{mM}$ Tris $\cdot \mathrm{HCl}, 1 \mathrm{mM}$ EGTA, $1 \mathrm{mM}$ EDTA, 1\% (vol/vol) Triton X-100, $0.27 \mathrm{M}$ sucrose, $10 \mathrm{mM}$ sodium 2-glycerophosphate, $1 \mathrm{mM}$ PMSF, $1 \mathrm{mM}$ benzamidine, $1 \mathrm{mM}$ sodium orthovanadate, 50 $\mathrm{mM} \mathrm{NaF}, 5 \mathrm{mM}$ sodium pyrophosphate, $20 \mathrm{mM}$ N-ethylmaleimide and complete protease inhibitors. The cell extracts were incubated for $4 \mathrm{~h}$ at $4{ }^{\circ} \mathrm{C}$ with Halo-TUBEs beads $(30 \mu \mathrm{L}$ packed volume). The beads were washed three times with lysis buffer containing $500 \mathrm{mM} \mathrm{NaCl}$ and once with $10 \mathrm{mM}$ Tris $\cdot \mathrm{HCl}, \mathrm{pH}$ 8.0. Proteins captured by TUBEs were released by denaturation in SDS and analyzed by immunoblotting.

\section{In vitro deubiquitination assay}


For preparation of ubiquitinated NICD1 as the substrate for the in vitro deubiquitination assay, HEK293T cells were transfected with $\mathrm{Ub}^{\mathrm{HA}}, \mathrm{NICD}^{\mathrm{V} 5}$ and FBXW7 ${ }^{\mathrm{FLAG}}$. After transfection for 36 hours, ubiquitinated NICD1 was purified from the cell extracts with anti-V5-agarose affinity beads (Sigma) in IPLS lysis buffer (50 mM Tris-HCL pH7.5, $120 \mathrm{mM} \mathrm{NaCl,} 0.5 \mathrm{mM}$ EDTA and 0.5\% Nonidet P-40) with 1x EDTA-Free Complete Protease Inhibitor Cocktail (Roche) and I $\mathrm{mM}$ phenylmethylsulfonyl fluoride. After extensive washing with the IPLS lysis buffer, the bound proteins were eluted with $500 \mu \mathrm{g} / \mathrm{ml}$ of V5-peptides (Sigma). For the in vitro deubiquitination assay, eluted ubiquitinated NICD1 protein was incubated with $0.25 \mu \mathrm{M}$ recombinant USP10 (LifeSensors) in a deubiquitination buffer (50mM Tris-HCl pH 8.0, 50mM $\mathrm{NaCl}, 10 \mathrm{mM}$ DTT, 5\% glycerol). As a positive control, $1 \mu \mathrm{M}$ USP2 catalytic core (6xHISUSP2-cc) was added. The assay reactions were incubated at $37^{\circ} \mathrm{C}$ for $1 \mathrm{~h}$. The reaction was terminated by adding Laemmli sample buffer containing 50 mM DTT. Ubiquitinated NICD1 was detected by Western blot analysis with anti-HA antibody. 6xHIS-USP2-cc was purified as previously described (11).

\section{Ub-Vs labeling}

For Ub-Vs labeling, HUVECs were lysed in Ub-Vs labeling buffer (50 mM HEPES pH7.4, 250 $\mathrm{mM}$ sucrose, $10 \mathrm{mM} \mathrm{MgCl} 2,2 \mathrm{mM}$ ATP, 1mM DTT) for $10 \mathrm{~min}$ on ice. Samples were cleared by centrifugation and protein concentrations determined by the Bradford procedure. Equal amounts of total protein lysates $(25 \mu \mathrm{g})$ were incubated with $0.5 \mu \mathrm{M}$ ubiquitin-vinyl sulfone (Ub-VS; U202; Boston Biochem) probe for $30 \mathrm{~min}$ at $37^{\circ} \mathrm{C}$. The samples were boiled in Laemmli sample buffer containing $50 \mathrm{mM}$ DTT at $95^{\circ} \mathrm{C}$ for $8 \mathrm{~min}$, separated by SDS-PAGE and transferred onto nitrocellulose membranes for further analysis by immunoblotting. 


\section{Animals and genetic experiments}

The conditional Usp10 knockout allele was generated by flanking exons 7 and 8 with loxP sites using standard strategies. Exons 7 and 8 encode for part of the USP (C19 peptidase) domain including the catalytic cysteine. Mice were on a C57BL/6 genetic background and were generated with genOway (Lyon, France). Constitutive Usp10 knockout mice were generated by crossing the floxed Usp10 mice with a CMV-driven cre deleter, which recombines in the germline (12). A detailed description of the knock-in mice overexpressing wild-type NICD1 (Rui Benedito) will be provided elsewhere. Briefly, mTomato-2A-nGFP-2A-NICD1-FLAG with a preceding floxed STOP cassette was knocked into the Rosa26 locus. Cre-mediated removal of the STOP sequence leads to CAG promoter-driven expression of the full-length NICD1, which also contains FLAG tag $\left(N I C D 1^{\mathrm{iEC}-\mathrm{OE}}\right)(13)$. In cre-recombined cells, NICD1 is released upon 2A-mediated cleavage alongside the reporters membrane-Tomato (mTOM) and nuclear-GFP (nGFP) (Fig. S7A). For inducible Cre-mediated recombination in ECs, floxed mice were bred with transgenic mice expressing the tamoxifen-inducible, Pdgfb promoter-driven creERT2 (14). For the analysis of angiogenesis in the postnatal mouse retina, cre-mediated recombination was induced in newborn mice by intraperitoneal (i.p.) injections of $25 \mu$ 4-hydroxy-tamoxifen (4OHT; $2 \mathrm{mg} / \mathrm{ml}$; Sigma) from postnatal day (P)1 - P4. Eyes were harvested at P6 or P7 for analysis. Control animals were littermate animals without cre expression. Mice were genotyped by PCR performed on genomic DNA. Genomic DNA was extracted from tail biopsies using the DirectPCR Lysis Reagent (Peqlab). Male and female mice were used for the analysis. All mice were maintained under specific pathogen-free conditions, and animal experiments were conducted in accordance with institutional guidelines. 


\section{Immunohistochemistry of murine retinal vasculature}

To analyse blood vessel growth in the postnatal retina, whole mouse eyes were fixed in $4 \%$ paraformaldehyde (PFA) on ice for $2 \mathrm{~h}$. Eyes were washed in phosphate-buffered saline (PBS) before the retinas were dissected and partially cut into 4 leaflets. After blocking / permeabilisation in 3\% fetal calf serum (FCS), 1\% BSA and 0.5\% Tween-20 (in PBS) for $1 \mathrm{~h}$ at room temperature, the retinas were incubated at $4{ }^{\circ} \mathrm{C}$ overnight in incubation buffer containing 1.5\% FCS, $0.5 \%$ BSA, $0.25 \%$ Tween-20 and $0.25 \%$ Triton X-100 (in PBS) and the primary antibody. Primary antibodies against the following proteins were used: Collagen IV (AbD Serotec, \#2150-1470, 1:400), ERG 1/2/3 (Abcam, \#ab92513, 1:200), ESM1 (R\&D Biosystems, \#AF1999, 1:100), ICAM2 (BD Biosciences, \#553326, 1:200), PECAM-1/CD31 (R\&D Biosystems, \#AF3628, 1:200). After 4 washes with 0.1\% Tween-20 in PBS (PBST), retinas were incubated with Alexa-Fluor 488- or Alexa-Fluor 647-conjugated secondary antibodies (Life Technologies, 1:400) for $2 \mathrm{~h}$ at room temperature. After 4 washes in PBST, retinas were flatmounted with ProLong Gold Antifade reagent (Life Technologies) and examined by confocal laser microscopy (Leica SP8). Immunostainings were carried out in tissues from littermates and processed under the same conditions. Volocity (Perkin Elmer), Fiji / ImageJ, Photoshop (Adobe) and Illustrator (Adobe) software were used for image acquisition and processing. For all of the images in which the levels of immunostaining were compared, settings for laser excitation and confocal scanner detection were kept constant between groups.

\section{Quantitative analysis of the retinal vasculature}


All quantifications were done on high-resolution confocal images of thin z-sections of the sample using the Volocity (Perkin Elmer) software. Endothelial coverage and the number of endothelial branch points were quantified behind the angiogenic front in a region between an artery and a vein. All parameters were quantified from a minimum of three vascularized fields per sample. Endothelial coverage was determined by assessing the ratio of the PECAM-positive area to the total area of the vascularized field (sized $200 \mu \mathrm{m} \times 200 \mu \mathrm{m}$ ), and expressed as a percentage of the area covered by PECAM-positive ECs. Average vein diameter was analyzed by assessing the diameter of individual veins calculated by averaging vein diameter measurements taken every $10 \mu \mathrm{m}$ along the entire length of the imaged vein. The number of sprouts and filopodial extensions were quantified at the angiogenic front. The total number of sprouts was normalized to an angiogenic front length of $100 \mu \mathrm{m}$, and the total number of filopodia was normalized to a vessel length of $100 \mu \mathrm{m}$ at the angiogenic front, which were defined and measured according to published protocols (15). The ESM1-positive area was measured in a vascularized field at the angiogenic front $(1500 \mu \mathrm{m} \times 350 \mu \mathrm{m})$ and further normalized to the PECAM-positive area in the same field. All of the images shown are representative of the vascular phenotype observed in samples from at least two distinct litters per group.

\section{Statistical analysis}

Statistical analysis was performed by unpaired, two-tailed Student's t-test, or non-parametric one-way ANOVA followed by Bonferroni's multiple comparison test using Prism 5 (GraphPad Software Inc.). For all bar graphs, data are represented as mean \pm S.E.M. P values $<0.05$ were considered significant. 


\section{Supplementary references}

1. V. Guarani et al., Acetylation-dependent regulation of endothelial Notch signalling by the SIRT1 deacetylase. Nature 473, 234-238 (2011).

2. J. Yuan, K. Luo, L. Zhang, J. C. Cheville, Z. Lou, USP10 regulates p53 localization and stability by deubiquitinating p53. Cell 140, 384-396 (2010).

3. C. Schulein-Volk et al., Dual regulation of Fbw7 function and oncogenic transformation by Usp28. Cell Rep 9, 1099-1109 (2014).

4. A. Shevchenko, H. Tomas, J. Havlis, J. V. Olsen, M. Mann, In-gel digestion for mass spectrometric characterization of proteins and proteomes. Nature Protocols 1, 2856-2860 (2006).

5. J. Rappsilber, Y. Ishihama, M. Mann, Stop and go extraction tips for matrix-assisted laser desorption/ionization, nanoelectrospray, and LC/MS sample pretreatment in proteomics. Analytical Chemistry 75, 663-670 (2003).

6. J. Cox, M. Mann, MaxQuant enables high peptide identification rates, individualized p.p.b.-range mass accuracies and proteome-wide protein quantification. Nature Biotechnology 26, 1367-1372 (2008).

7. J. Cox et al., Andromeda: a peptide search engine integrated into the MaxQuant environment. Journal of Proteome Research 10, 1794-1805 (2011).

8. T. Zhang et al., Prmt5 is a regulator of muscle stem cell expansion in adult mice. Nat Commun 6, 7140 (2015).

9. R. Hjerpe et al., Efficient protection and isolation of ubiquitylated proteins using tandem ubiquitin-binding entities. EMBO Reports 10, 1250-1258 (2009).

10. C. H. Emmerich et al., Activation of the canonical IKK complex by K63/M1-linked hybrid ubiquitin chains. Proc Natl Acad Sci U S A 110, 15247-15252 (2013).

11. R. T. Baker et al., Using deubiquitylating enzymes as research tools. Methods in Enzymology 398, 540-554 (2005).

12. H. Su, A. A. Mills, X. Wang, A. Bradley, A targeted X-linked CMV-Cre line. Genesis (New York, N.Y. : 2000) 32, 187-188 (2002).

13. S. Pontes-Quero et al., Dual ifgMosaic: A Versatile Method for Multispectral and Combinatorial Mosaic Gene-Function Analysis. Cell 170, 800-814.e818 (2017).

14. S. Claxton et al., Efficient, inducible Cre-recombinase activation in vascular endothelium. Genesis (New York, N.Y. : 2000) 46, 74-80 (2008).

15. M. E. Pitulescu, I. Schmidt, R. Benedito, R. H. Adams, Inducible gene targeting in the neonatal vasculature and analysis of retinal angiogenesis in mice. Nature Protocols $\mathbf{5}$, 1518-1534 (2010). 
Fig. S1. USP10 is a robustly expressed deubiquitinase in endothelial cells.

A, Schematic illustration of the SILAC-based quantitative proteomic screen. B, RNA-seq analysis of RNA purified from HUVECs comparing the expression profile of USP family members. C, RNA-sequencing (RNA-seq) analysis of HUVECs comparing USP10 expression with those of several endothelial genes. D, mRNA expression of USP10 in different subtypes of human ECs as determined by RNA-seq analysis. HAEC, human aortic endothelial cells. HMVEC, human micro-vascular endothelial cells. HLEC, human lymphatic endothelial cells. E, Co-immunoprecipitation of NICD1 and USP10 from cytoplasmic (Cyto) and nuclear (Nuc) fractions derived from HUVECs treated with or without DLL4 for $24 \mathrm{~h}$. NICD1 was immunoprecipitated by anti-NOTCH1 antibody. Error bars: mean \pm SEM of $n=3$. HUVEC, human umbilical vein endothelial cells.

\section{Fig. S2. Regulation of DLL4-Notch target genes by USP10 in ECs.}

A, Volcano plot comparison of gene expression patterns between siCtrl and siUSP1O transfected HUVECs demonstrating the selectivity of the USP10-directed RNA interference approach. The $\mathrm{x}$ axis depicts the differential expression profiles, plotting the fold-induction ratios in a $\log _{2}$ scale. The $y$ axis depicts the statistical significance in expression ( $\mathrm{P}$ value from a t-test) in a $\log _{10}$ scale. Deubiquitinase genes are indicated in orange while the rest of the genes are indicated in grey. B, Activation of HES1-driven luciferase (luc) reporter in siCtrl- or siUSP10-transfected HUVECs after treatment with vehicle or DLL4, in the presence or absence of a $\gamma$-secretase inhibitor (DBZ) for $24 \mathrm{~h}$. C, Heat map of differentially expressed genes in siCtrl- or siUSP10-transfected HUVECs treated with vehicle or DLL4 for $24 \mathrm{~h}$. Genes with a cut-off $\mathrm{P}$ value of $<0.05$ and a 
fold-change of $<0.5$ between siCtrl and siCtrl + DLL4 are shown. D, Quantitative RT-PCR (qRT-PCR) analysis of a selected panel of DLL4-regulated genes in siCtrl- or siUSP10transfected HUVECs treated with vehicle or DLL4 for $24 \mathrm{~h}$. Values are represented relative to vehicle treated control after normalization to $\beta$-ACTIN expression. E, qRT-PCR analysis for HEY2 mRNA expression from HUVECs harvested at 6-hour intervals over $48 \mathrm{~h}$ of DLL4 treatment. Values are represented relative to expression of $\beta$-ACTIN. F, mRNA expression of Notch pathway components in un-stimulated siCtrl or siUSP10 HUVECs $24 \mathrm{~h}$ post transfection. Error bars: mean \pm SEM of $\mathrm{n}>3 . * P<0.05, * * P<0.01$, $* * * P<0.001$, $* * * * P<0.0001$, NS, not significant.

\section{Fig. S3. USP10 inactivation reduces NICD1 protein levels in ECs.}

A, Quantification of NICD1 levels in siCtrl- or siUSP10-transfected HUVECs stimulated with DLL4 or vehicle for $24 \mathrm{~h}$. B, Immunoblot blot analysis of NICD1 expression in solvent or DLL4-treated HUVECs transfected with control (siCtrl) or different USP10 siRNAs (siUSP101/2). C, Quantification of NICD1 levels in control (gCtrl) and USP10 knockout ECs (gUSP10) generated by lentiviral transduction of HUVECs with control or USP10-targeting gRNAs as well as a CAS9 nuclease. HUVECs were stimulated with vehicle or DLL4 for $24 \mathrm{~h}$. D, NOTCH1 mRNA expression in gCtrl and gUSP10 ECs. NOTCH1 expression was determined by quantitative RT-PCR and values are represented relative to $\beta$-ACTIN expression. $\mathbf{E}$, Immunoblot

analysis of NICD1 expression in siCtrl- or siUSP10-transfected HUVECs stimulated with equal concentrations $(500 \mathrm{ng} / \mathrm{ml})$ of DLL1, DLL4 or JAG1 for 6 and 24 h. F,G, NICD1 levels in siCtrl- and siUSP10-transfected HUVECs treated with increasing concentrations (up to 2.5 
$\mu \mathrm{g} / \mathrm{ml})$ of DLL1 $(\mathbf{F})$ or JAG1 $(\mathbf{G})$. Error bars: mean \pm SEM of $\mathrm{n}>3$. Error bars: mean \pm SEM of $\mathrm{n}>3 . * * \mathrm{P}<0.01$, NS, not significant.

\section{Fig. S4. USP10, but not other USP family members, increases NICD1 abundance in ECs.}

A, Immunoblot blot analysis of NICD1 protein levels in total cell lysates harvested from HUVECs transfected with control, USP10, USP7, USP11 or USP15 siRNA followed by vehicle or DLL4 treatment for $24 \mathrm{~h}$. B, NICD1 ${ }^{\mathrm{V} 5}$ immunoblot analysis from HUVECs transfected with siCtrl or siUSP10, followed by transduction with a control or NICD $1^{\mathrm{V} 5}$-encoding adenovirus. NICD $1^{\mathrm{V} 5}$ levels were determined after $24 \mathrm{~h} . \mathbf{C}$, NICD1 expression in total cell lysates obtained from HUVECs transduced with a control or USP10-FLAG encoding adenovirus followed by vehicle or DLL4 treatment for $24 \mathrm{~h}$.

\section{Fig. S5. USP10 regulates the ubiquitin-dependent degradation of NICD1.}

A, NICD1 protein levels in DLL4-treated HUVECs transfected with siCtrl, siUSP10, siFBXW7 or combinations thereof. NICD1 expression was determined at $24 \mathrm{~h}$. B, Immunoblot analysis of NICD1 expression in siCtrl and siUSP10-transfected HUVECs treated with vehicle, DLL4, the proteasome inhibitor MG132 or combinations thereof. The analysis was performed $24 \mathrm{~h}$ post DLL4 stimulation. C, Immunoblot blot analysis for p53 and SIRT6 protein expression in HUVECs transfected with siCtrl or siUSP10. D, Ubiquitination of endogenous NICD1 HUVECs stimulated with vehicle or DLL4 for $24 \mathrm{~h}$. MG132 was added to some of the samples for the last 6 hours of the stimulation. Cell lysates were incubated with Halo-tagged tandem ubiquitin binding entities ( ${ }^{\text {Halo }}$ TUBE) to enrich ubiquitinated proteins. Ubiquitinated NICD1 was detected 
by immunoblotting using an NICD1-specific antibody. E, Assessment of NICD1 ubiquitination in HUVECs transduced with a control- (LentiCtrl) or USP10-encoding lentivirus (LentiUSP10 ${ }^{\mathrm{GFP}}$ ) stimulated with vehicle or DLL4 for $24 \mathrm{~h}$. F, Detection of polyubiquitinated NICD1 ${ }^{\mathrm{V} 5}$ in HEK293T cells that were transfected with V5-tagged NICD1 (NICD1 ${ }^{\mathrm{V} 5}$ ), FLAGtagged FBXW7 $\left(\mathrm{FBXW} 7^{\mathrm{FLAG}}\right)$ and HA-tagged ubiquitin $\left(\mathrm{Ub}^{\mathrm{HA}}\right)$. Total cell lysates were harvested, denatured and then immunoprecipitated using an anti-V5 antibody followed by Western blotting for HA.

\section{Fig. S6. Generation and phenotypes of Usp10 mutant mice.}

A, Schematic illustration of the strategy used to generate a conditional Usp10 mutant allele in which exons 7 and 8 are flanked by $\operatorname{lox} P$ sites (red triangles). The assembly of the genomic locus, the targeted allele and the flp- and cre-recombined loci are shown. FRT-Neo-FRT, neomycin resistance cassette flanked by FRT sites. B, Table of genotypes from $U s p 10^{+/}$(male) and $U s p 10^{+-}$(female) intercrosses. No viable $U s p 10^{-/}$mice were detected at birth. C, PCR analysis of genomic DNA from control $\left(U s p 10^{+/+}\right.$, lane $1 ; U s p 10^{f l /+}$, lane $2 ; U s p 10^{f l f l}$, lanes 3 and 5) and Usp10 $0^{i E C-K O}$ (Pdgfb-creERT2;Usp10 $0^{f l f l}$, lanes 4 and 6) pups untreated (lanes 1 to 4) or treated (lanes 5 and 6) with 4-OHT. Recombination of the floxed Usp10 allele ( $\Delta$ ) occurs only in 4-OHT-injected animals that carry the Pdgfb-creERT2 transgene. D, Higher magnification images and quantifications of the ESM1 immunostaining (yellow) in the retinal vasculature of control and Usp $10^{i E C-K O}$ mutants that were also labeled for PECAM (grey) and ERG (red). The lower panels show the isolated ESM1 signal in grey. E, Morphology of arteries and veins in retinas of control and $U s p 10^{i E C-K O}$ at P7. Retinas were labeled with PECAM (blue), ICAM (ICAM2; green) and collagen IV (COL; red) antibodies. The quantification of the average vein 
diameter is shown on the right. Error bars: mean \pm SEM of $\mathrm{n}>3 . * P<0.05, * * P<0.01$, $* * * * P<0.0001$,

\section{Fig. S7. Inducible overexpression of NICD1 in ECs of mice causes vascular rarefaction.}

A, A cassette containing the CAG promoter, a floxed STOP sequence, and a cDNA encoding for membrane-bound Tomato, nuclear GFP and NICD1-FLAG was inserted into the Rosa26 locus. 2A autocleavage sites flank the middle gene protein product (i.e., nuclear GFP). A schematic representation of the wild-type Rosa26 locus, the floxed allele, and the recombined allele after

cre expression is shown. $\mathbf{B}$, Confocal images of control and $N I C D 1^{i E C-O E}$ mice that were injected with 4-OHT from P1 to P4 and analysed for PECAM (blue), membrane-bound tomato (TOM; red) and nuclear GFP (green) expression at P6. The lower panels show the isolated PECAM signal in grey. C, Immunofluorescence staining for ERG (cyan) and PECAM (red) in a P6 mouse retina of 4-OHT-injected control and $N I C D I^{i E C-O E}$ mice. D, Quantifications of vascular parameters in control and $N I C D 1^{i E C-O E}$ mice as indicated. Error bars: mean $\pm \mathrm{SEM}$ of $\mathrm{n}>3$. Error bars: mean \pm SEM of $\mathrm{n}>3 . * * * \mathrm{P}<0.001, * * * * \mathrm{P}<0.0001$

Fig. S8. USP10 activity is increased by hypoxia and starvation in ECs.

A,B, Nutrient deprivation or hypoxia regulates USP10 activity in cultured ECs. To assess USP10 activity, a modified deubiquitinating enzyme substrate, ubiquitin vinyl sulfone (Ub-VS), was used. Upon catalysis of Ub-VS, a covalent bond is formed between the substrate and the active cysteine residue of the enzyme, leading to a mobility shift of $8 \mathrm{kDa}$ of the active enzyme that can be detected by immunoblotting. HUVECs were cultured under normal, starved ( 1 h) (A) or 
hypoxic conditions $\left(6 \mathrm{~h}, 0.2 \% \mathrm{O}_{2}\right)(\mathbf{B})$ and then lysed for labeling with a Ub-VS probe. The protein lysates were then subjected to immunoblotting with an USP10 antibody. 